\title{
TOPOLOGY OF THE UNIVERSE: THEORY AND OBSERVATION
}

\author{
JEAN-PIERRE LUMINET ${ }^{1}$ AND BOUDEWIJN F. ROUKEMA ${ }^{2,3}$ \\ 1 DARC, Observatoire de Paris-Meudon, 5 place Jules Janssen, \\ F-92195 Meudon Cedex, France (Jean-Pierre.Luminet@obspm.fr) \\ AND \\ ${ }^{2}$ Institut d'Astrophysique de Paris, 98bis Bd Arago, F-75.014, Paris, France \\ ${ }^{3}$ Inter-University Centre for Astronomy and Astrophysics, Post Bag 4 \\ Ganeshkhind, Pune, 411 00\%, India (boud@iucaa.ernet.in)
}

\begin{abstract}
One could imagine that as a result of enormously extended astronomical experience, the entire universe consists of countless identical copies of our Milky Way, that the infinite space can be partitioned into cubes each containing an exactly identical copy of our Milky Way. Would we really cling on to the assumption of infinitely many identical repetitions of the same world? ... We would be much happier with the view that these repetitions are illusory, that in reality space has peculiar connection properties so that if we leave any one cube through a side, then we immediately reenter it through the opposite side." (Schwarzschild 1900, translation 1998)

Developments in the theoretical and observational sides of cosmic topology were slow for most of the century, but are now progressing rapidly, at the scale of most interest which is $1-10 h^{-1}$ Gpc rather than $10 \mathrm{kpc}$.

The historical, mathematical and observational sides of this subject are briefly reviewed in this course.
\end{abstract}

\section{Introduction}

Think of a right triangle drawn on a transparency. The transparency is a piece of an infinite Euclidean plane whose flat metric is expressed by Pythagoras' Theorem. Bend the transparency around into a cylinder. The latter is thus obtained by identifying two opposite edges of the transparency. The new surface is locally flat (Pythagoras' Theorem stills holds perfectly), but it has a quite different shape. It contains closed geodesics. Next we can identify the two remaining edges and get a flat torus (this is a thought experiment, because the usual torus, or "annulus", visualized as a surface of revolution in 3-dimensional Euclidean space, has positive Gaussian curvature at some places and negative at others). The flat torus has still a locally Euclidean metric although its global shape has drastically changed: it is a finite surface without borders.

These elementary operations well illustrate the difference between the curvature (given by the metric) and the topology. Both are needed in order to know the full geometry of a twodimensional, or more importantly, a three-dimensional space. This applies as well to physical space, namely to cosmological models for describing the Universe as a whole. The shape of space is a fundamental issue in physics. Curiously enough, this Cargese '98 summer school on cosmology is probably the first one to include a couple of lectures on cosmic topology. As we 
shall see below, both for historical and for practical reasons, cosmic topology has been widely ignored during 80 years of relativistic cosmology, except by some pioneering authors.

After an historical section 2, sections 3-4 are mostly based on the review paper by [45] (hereafter LaLu 95). Since the mid 90's, worldwide interest for cosmological topology has blossomed, from a mathematical point of view, mostly related to progress in the understanding of compact hyperbolic manifolds, from a theoretical point of view, as well as from an observational point of view, related to improvements of data on the large scale 3-dimensional distribution of cosmic objects and on the 2-dimensional structure of the cosmic microwave background. Since these lectures are aimed to provide a general view of the subject, only some of the new mathematical results (obtained since the 1995 revival) will be mentioned. An incomplete list of theoretical articles related to the possible physical origin of cosmic topology is provided towards the end of section 2. For the latest developments in all aspects of the subject, see the proceedings of the 1997 and 1998 workshops [76, 67]. Section 5 of this lecture, devoted to the observational aspects of cosmic topology, provides an up-date and complement to the review of [69].

\section{A Brief History of Cosmological Topology}

Most of this historical introduction is taken from [55], see also [75].

Is space finite or infinite, oriented or not, made of one piece or not, has it holes or handles, what is its global shape? A common misconception is to believe that Einstein's general relativity theory is all one needs to answer such paramount questions. However, general relativity deals only with local geometrical properties of the universe, such as its curvature, not with its global characteristics, namely its topology.

The physical extension of space is one of the oldest cosmological questions, going back to twenty-five centuries of cosmological modelling (e.g. [56]). In the history of cosmology, it is well known that Newtonian physical space, mathematically identified with the infinite Euclidean space $\mathbf{R}^{3}$, gave rise to paradoxes such as the darkness of the night sky (see e.g. [38]) and to problems of boundary conditions.

Wondering about absolute accelerations, Newton imagined a bucket swung on the end of a rope and compared it with a bucket at rest. If the bucket is at rest, the surface of the water remains flat. If the bucket rotates, the water surface becomes concave. Newton argued that the concave shape of the water could not be due to its relative motion with respect to the bucket, and that it proved the existence of an absolute centrifugal acceleration. Mach considered the same problem and criticised Newton's reasoning [2]: what we observe is that the bucket rotates with respect to the fixed stars, but, who is to say whether the bucket is really rotating with respect to the stars at rest or whether the stars are really rotating with respect to the bucket? According to Newton, we should observe the concavity in the first case and not in the second. This is because Newton assumed an absolute frame related to the global distribution of matter in the universe (the fixed stars). Mach denied the concept of absolute acceleration. According to him, a rotating body in a non-rotating universe or a non-rotating body in a rotating universe should give the same result: centrifugal force. Mach concluded that the inertial mass of a body should result from the contributions of all the masses in the universe. But an obvious divergence difficulty arose, since a homogeneous Newtonian universe with non-zero density has an infinite mass. Hence, Mach supported the idea of a finite universe in order to have a finite local inertia.

By the end of the $X I X^{t h}$ century, mathematicians such as Riemann had discovered a variety of finite spaces without boundaries, such as the hypersphere and the projective space. Schwarzschild [72] brought this work to the attention of the astronomical community in 1900, and he considered the possibility that the space could have a non-trivial topology: "One could imagine that as a result of enormously extended astronomical experience, the entire universe consists of countless identical copies of our Milky Way, that the infinite space can be partitioned 
into cubes each containing an exactly identical copy of our Milky Way. Would we really cling on to the assumption of infinitely many identical repetitions of the same world? ... We would be much happier with the view that these repetitions are illusory, that in reality space has peculiar connection properties so that if we leave any one cube through a side, then we immediately reenter it through the opposite side" (English translation [73]).

In modern terms, Schwarzschild told us that if the galaxies were found to lie in a rectangular lattice, with images of the same galaxy repeating at equivalent lattice points, then we could conclude that the Universe is a 3 -torus.

Cosmology developed rapidly after Einstein's 1915 discovery of general relativity. General relativity explains gravity as the curvature of spacetime; the latter is determined by the density of matter-energy. The aim of relativistic cosmology is to deduce from the gravitational field equations some physical models of the Universe as a whole. When Einstein (1917) assumed in his static cosmological solution that space was a positively-curved hypersphere, one of his strongest motivations was to provide a model for a finite space, although without a boundary. Following Mach and Riemann, he regarded the closure of space as necessary to solve the problem of inertia and chose the hypersphere as his model of space.

The Einstein space model cleared up most of the paradoxes stemming from Newtonian cosmology in such an elegant way that most cosmologists of the time adopted the new paradigm of a closed space, without examining other geometrical possibilities. Einstein was also convinced that the hypershere provided not only the metric of cosmic space - namely its local geometrical properties - but also its global structure, its topology. However, topology does not seem to have been a major preoccupation of Einstein; his 1917 cosmological article did not mention any topological alternative to the spherical space model.

Some of his colleagues pointed out to Einstein the arbitrariness of his choice. Indeed the global shape of space is not only depending on the metric; it depends primarily on its topology, and requires a complemenraty approach to Riemannian differential geometry. Since Einstein's equations are partial derivative equations, they describe only local geometrical properties of spacetime. The latter are contained in the metric tensor, which enables us to calculate the components of the curvature tensor at any non-singular point of spacetime. But Einstein's equations do not fix the global structure of spacetime: to a given metric solution of the field equations, correspond several (and in most cases an infinite number of) topologically distinct universe models.

Firstly, de Sitter [14] noticed that the Einstein's solution admitted a different spaceform, the 3-dimensional projective space (also called elliptic space), obtained from the hypersphere by identifying antipodal points. The projective space has the same metric as the spherical one, but a different topology (for instance, for the same curvature radius its volume is half that of the spherical space).

H. Weyl also pointed out the freedom of choice between spherical and elliptical topologies. Einstein's answer [18] was unequivocal: "Nevertheless I have like an obscure feeling which leads me to prefer the spherical model. I have a feeling that manifolds in which any closed curve can be continuously contracted to a point are the simplest ones. Other persons must share this feeling, otherwise astronomers would have taken into account the case where our space is Euclidean and finite. Then the two-dimensional Euclidean space would have the connectivity properties of a ring surface. It is an Euclidean plane in which any phenomenon is doubly periodic, where points located in the same periodical grid are identical. In finite Euclidean space, three classes of non continuously contractible loops would exist. In a similar way, the elliptical space possesses a class of non continuously contractible loops, contrary to the spherical case; it is the reason why I like it less than the spherical space. Can it be proved that elliptical space is the only variant of spherical space? It seems yes to me". 
Einstein [19] repeated his argumentation in a postcard sent to Felix Klein: "I would like to give you a reason why the spherical case should be preferred to the elliptical case. In spherical space, any closed curve can be continuously contracted to a point, but not in the elliptical space; in other words the spherical space alone is simply-connected, not the elliptical one [...] Finite spaces of arbitrary volume with the Euclidean metric element undoubtedly exist, which can be obtained from infinite spaces by assuming a triple periodicity, namely identity between sets of points. However such possibilities, which are not taken into account by general relativity, have the wrong property to be multiply-connected'.

From these remarks it seems that the Einstein's preference for simple-connectedness of space was of an aesthetical nature, rather than being based on physical reasoning.

In his answer to Weyl, Einstein was definitely wrong on the last point: in dimension three, an infinite number of topological variants of the spherical space - all closed - do exist, including the so-called lens spaces (whereas in dimension two, only two spherical spaceforms exist, the ordinary sphere and the elliptic plane). However, nobody knew this result in the 1920's: the topological classification of 3-dimensional spaces was still at its beginnings. The study of Euclidean spaceforms started in the context of crystallography. Feodoroff [30] classified the 18 symmetry groups of crystalline structures in $\mathbf{R}^{3}$, Bieberbach [4] developed a full theory of crystallographic groups, and it took twenty years before Novacki [59] showed how Bieberbach's results could be applied to complete the classification of 3-dimensional Euclidean spaceforms. The case of spherical spaceforms was first treated by Klein [43] and Killing [44]. The problem was fully solved much later [87]. Eventually, the classification of homogeneous hyperbolic spaces shot forward in the 1970's; it is now an open field of intensive mathematical research [79, 80].

Returning to relativistic cosmology, the discovery of non-static solutions by Friedmann [32] and, independently, Lemaître [48], opened a new era for models of the universe as a whole (see, e.g., [54] for an epistemological analysis). Although Friedmann and Lemaître are generally considered as the discoverers of the big bang concept - at least of the notion of a dynamical universe evolving from an initial singularity - one of their most original considerations, devoted to the topology of space, was overlooked. As they stated, the homogeneous isotropic universe models (F-L models) admit spherical, Euclidean or hyperbolic spacelike sections according to the sign of their (constant) curvature (respectively positive, zero or negative). In addition, Friedmann [33] pointed out the topological indeterminacy of the solutions in his popular book on general relativity, and he emphasized how Einstein's theory was unable to deal with the global structure of spacetime. He gave the simple example of the cylinder. Generalizing the argument to higher dimensions, he concluded that several topological spaces could be used to describe the same solution of Einstein's equations.

Topological considerations were fully developed in his second cosmological article, although primarily devoted to the analysis of hyperbolic solutions. Friedmann [34] clearly outlined the fundamental limitations of relativistic cosmology: "Without additional assumptions, Einstein's world equations do not answer the question of the finiteness of our universe", he wrote. Then he described how space could be finite (and multi-connected) by suitably identifying points. He also predicted the possible existence of "ghost" images of astronomical sources, since at the same point of a multi-connected space an object and its ghosts would coexist. He added that " $a$ space with positive curvature is always finite", but he recognized the fact that the mathematical knowledge of his time did not allow him to "solve the question of finiteness for a negativelycurved space".

By contrast with Einstein's reasoning, it is seen that the Russian cosmologist had no prejudice in favour of a simply-connected topology. Certainly, Friedmann believed that only finite volume spaces were physically realistic. Prior to his discovery of hyperbolic solutions, the cosmological solutions derived by Einstein, de Sitter and himself had a positive spatial curvature, thus a finite volume. With negatively-curved spaces, the situation became problematic, because the "natural" 
topology of hyperbolic space has an infinite volume. It is the reason why Friedmann, in order to justify the physical pertinence of his solutions, emphasized the possibility of compactifying space by suitable identifications of points.

Lemaitre fully shared the common belief in the closure of space. He expressed his view that Riemannian geometry "dissipated the nightmare of infinite space" [51]. Thus Lemaitre [48, 49] assumed positive space curvature, but he thoroughly discussed the possibility of projective space, that he preferred to the spherical one. Later, he [50] also noticed the possibility of hyperbolic and Euclidean spaces with finite volumes for describing the physical universe.

Such fruitful ideas of cosmic topology remained widely ignored by the main stream of big bang cosmology. Perhaps the Einstein-de Sitter [20] model, which assumed Euclidean space and eluded the topological question, had a negative influence on the development of the field. Almost all subsequent textbooks and monographs on relativistic cosmology assumed that the global structure of the universe was either the finite hypersphere, or the infinite Euclidean space, or the infinite hyperbolic space, without mentioning at all the topological indeterminacy. As a consequence, some confusion settled down about the real meaning of the terms "open" and "closed" used to characterize the F-L solutions. Whereas they apply correctly to time evolution (open models stand for ever-expanding universes, closed models stand for expandingcontracting solutions), they do not properly describe the extension of space (open for infinite, closed for finite). Nevertheless it is still frequent to read that the (closed) spherical model has a finite volume whereas the (open) Euclidean and hyperbolic models have infinite volumes. The correspondance is true only in the very special case of a simply-connected topology and a zero cosmological constant. According to Friedmann's original remark, in order to know if a space is finite or infinite, it is not sufficient to determine the sign of its spatial curvature, or equivalently in a cosmological context to measure the ratio $\Omega_{0}$ of the average density to the critical value: additional assumptions are necessary — those arising from topology, precisely.

The idea of a multi-connected universe has never been entirely forgotten. In a very comprehensive article, Ellis [21] detailed the classification of 3-dimensional Riemannian manifolds useful for cosmology and started to explore the observational consequences of a toroidal universe. Others, such as Sokoloff and Schvartsman, Fang and Sato, Gott, Fagundes, investigated flat and non-flat spaces (see complete references in LaLu95). Nevertheless, until 1995, investigations in cosmic topology were rather scarce. From an epistemological point of view, it seems that the prejudice in favour of simply-connected (rather than multi-connected) spaces was of the same kind as the prejudice in favour of static (rather than dynamical) cosmologies during the 1920's. At a first glance, "Occam's razor" (often useful in physical modelling) could be invoked to preferably select the simply-connected topologies. However, on the theoretical side, new approaches to spacetime, such as quantum cosmology, suggest that the smallest closed hyperbolic manifolds are favoured, thus providing a new paradigm for what is the "simplest" manifold. On the observational side, present astronomical data (e.g. [12]) indicate that the average density of the observable universe is less than the critical value $\left(\Omega_{0}=0.3-0.4\right)$, thus suggesting that we live in a negatively-curved F-L universe (unless the cosmological constant is positive and large enough). Putting together theory and observation, cosmologists must face the fact that a negatively-curved space can have a finite volume, and that in that case it must be multi-connected.

In the last few decades, much effort in observational and theoretical cosmology has been directed towards determining the curvature of the universe. The problem of the topology of spacetime was generally ignored within the framework of classical relativistic cosmology. It began to be taken seriously discussed in quantum gravity for various reasons: the spontaneous birth of the Universe from a quantum vacuum requires the Universe to have compact spacelike hypersurfaces, and the closure of space is a necessary condition to render tractable the integrals of quantum gravity [1]. 
Further work in quantum gravity and particle physics which explores ideas of space-time topology globally and/or as the basis of a quantum gravity theory includes that of $[39,88,57$, $7,41,16,15,64,17,74]$.

However, the topology of spacetime also enters in a fundamental way in classical general relativity. Many cosmologists were surprisingly unaware of how topology and cosmology could fit together to provide new insights in universe models. Aimed to create a new interest in the field of cosmic topology, the review by LaLu95 stressed what multi-connectedness of the Universe would mean and on its observational consequences. However two different papers ([78] ; [13]) declared that the small universe idea was "no longer an interesting cosmological model". However, the authors drew very general conclusions from considering only a few special 3-manifolds in the Euclidean case, and by adopting assumptions on the primordial perturbation spectrum which are observationally justified on large scales only by assuming simple connectedness. They did not take into account a very interesting class of realistic universe models, namely the compact hyperbolic manifolds, which require a quite different treatment. Ironically, approximately the same amount of papers in cosmic topology have been published within the last 3 years as in the previous 80 years!

\section{Mathematical background}

\subsection{BASICS OF TOPOLOGY}

The topological properties of a manifold are those which remain insensitive to continuous transformations. Thus, size and distance are in some sense ignored in topology: stretching, squeezing or "kneading" a manifold change the metric but not the topology; cutting, tearing or making holes and handles change the latter. It is often possible to visualize two-dimensional manifolds by representing them as embedded in three-dimensional Euclidean space (such a mapping does not necessarily exist however, e.g. the Klein bottle and the flat torus). Three-dimensional manifolds require the introduction of more abstract representations, using, for example, the fundamental domain.

The different topological surfaces can be represented by polygons whose edges are suitably identified by pairs. As we have seen in the introduction, identifying one pair of opposite edges of a square gives a portion of a cylinder; then, stretching the cylinder and bending it around in such a way that one can glue together the two circular ends generates a simple torus, a closed surface (figure 1). The torus is thus topologically equivalent to a rectangle with opposite edges identified. The rectangle is called a fundamental domain of the torus. From a topological point of view (namely without reference to size), the fundamental domain can be chosen in different ways: a square, a rectangle, a parallelogram, even a hexagon (since the plane can be tiled by hexagons, the flat torus can be also represented by a hexagon with suitable identification of edges).

The gluing method described above becomes extremely fruitful when the surfaces are more complicated. A two-dimensional g-torus $T_{g}$ is a torus with $g$ holes. $T_{g}$ can be constructed as the connected sum of $g$ simple tori (figure 2). The g-torus is therefore topologically equivalent to a connected sum of $g$ squares whose opposite edges have been identified. This sum is itself topologically equivalent to a $4 \mathrm{~g}$-gon where all the vertices are identical with each other and the sides are suitably identified by pairs.

It would be tempting to visualize the g-torus by gluing together equivalent edges, like for the simple torus. But such an operation is not straightforward when $g \geq 2$. All the vertices of the polygon correspond to the same point of the surface. Since the polygon has at least 8 edges, it is necessary to make the internal angles thinner in order to fit them suitably around a single vertex. This can only be achieved if the polygon is represented in the hyperbolic plane $\mathbf{H}^{2}$ instead of the Euclidean plane $\mathbf{R}^{2}$ : this increases the area and decreases the angles. The 

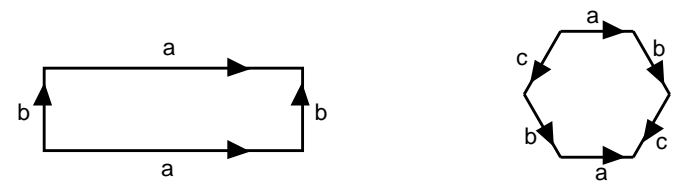

$\sqrt{n}$

$\sqrt{1}$
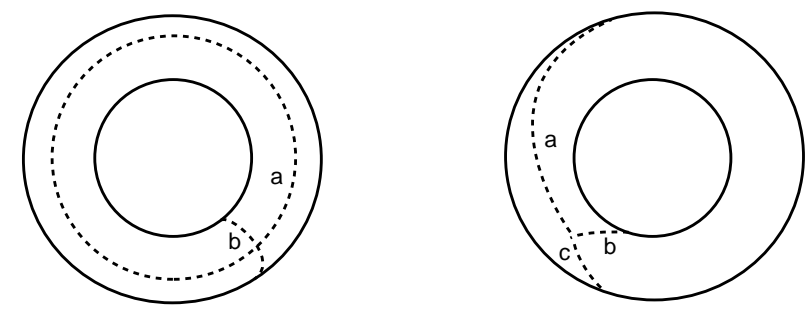

Figure 1. Construction of the flat torus from a rectangle and from an hexagon with suitable identifications
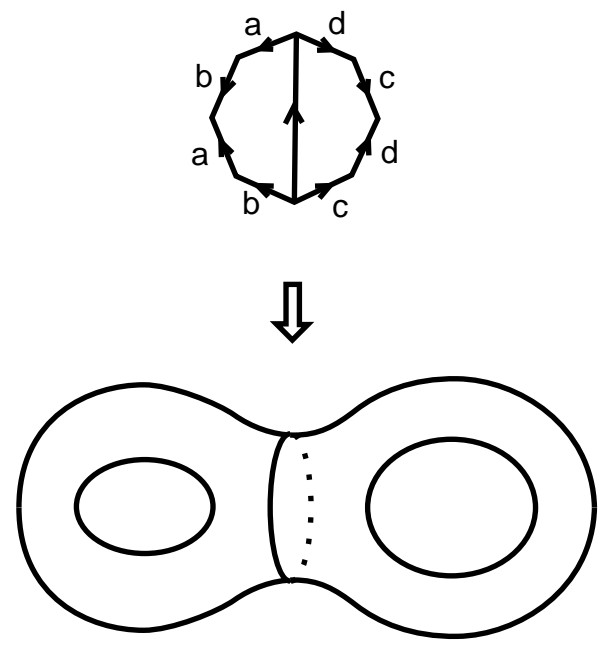

Figure 2. The 2-torus as the connected sum of two simple tori

more angles to fit together, the thinner they have to be and the greater the surface. The g-torus $(g \geq 2)$ is therefore a compact surface of negative curvature.

When one deals with more than two dimensions, the gluing method remains the simplest way to visualize spaces. By analogy with the two-dimensional case, the three-dimensional simple torus (also referred to as the hypertorus) is obtained by identifying the opposite faces of a parallelepiped. The resulting volume is finite. Let us imagine a light source at our position, immersed in such a structure. Light emitted backwards crosses the face of the parallelepiped behind us and reappears on the opposite face in front of us; therefore, looking forward we can see our back (as in the spherical universe model of Einstein). Similarly, we see in our right our left profile, or upwards the bottom of our feet. In fact, for light emitted isotropically, and for an arbitrarily large time to wait, we could observe ghost images of any object viewed arbitrarily close to any angle. The resulting visual effect would be comparable (although not identical) to what could be seen from inside a parallelepiped of which the internal faces are covered with mirrors. Thus one would have the visual impression of infinite space, although the real space is closed. More generally, any three dimensional compact manifold can be represented as a polyhedron what we define later more precisely as the fundamental domain (hereafter $F D$ ) - whose faces 


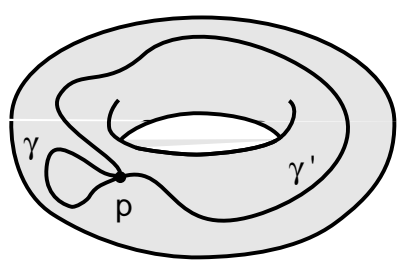

Figure 3. Classes of homotopy of a torus $\mathbf{S}^{1} \times \mathbf{S}^{1}$. Loops can wind $\mathrm{m}$ times around the central hole and $\mathrm{n}$ times around the body of the torus. Thus the fundamental group consists of pairs $(m, n)$ of integers with addition $(m, n)+(p, q)=(m+p, n+q)$. In other words it is isomorphic to $\mathbf{Z} \oplus \mathbf{Z}$.

are suitably identified by pairs. But, as soon as the number of faces of the $F D$ exceeds 8 , the compact manifold resulting from identifications cannot be developed into the Euclidean space $\mathbf{R}^{3}$ : the $F D$ must be built in hyperbolic space $\mathbf{H}^{3}$ in order to fit all the angles together at the vertices.

The general method for classifying the topologies of a given manifold $\mathcal{M}$ is:

- to determine its universal covering space $\widetilde{\mathcal{M}}$

- to find the fundamental domain FD

- to calculate the holonomy group acting on the $F D$.

All these concepts have very formal and abstract definitions that can be found in classical textbooks in topology. We introduce below basic definitions.

The strategy for characterizing spaces is to produce invariants which capture the key features of the topology and uniquely specify each equivalence class. The topological invariants can take many forms. They can be just numbers, such as the dimension of the manifold, the degree of connectedness or the Poincaré - Euler characteristic. They can also be whole mathematical structures, such as the homotopy groups.

A loop at $\mathbf{x} \in \mathcal{M}$ is any path which starts at $\mathbf{x}$ and ends at $\mathbf{x}$. Two loops $\gamma$ and $\gamma^{\prime}$ are homotopic if $\gamma$ can be continuously deformed into $\gamma^{\prime}$. The manifold $\mathcal{M}$ is simply-connected if, for any $\mathbf{x}$, two any loops through $\mathbf{x}$ are homotopic. Equivalently, it is simply-connected if every loop is homotopic to a point. If not, the manifold is said to be multi-connected. Obviously, the Euclidean spaces $\mathbf{R}^{1}, \mathbf{R}^{2}, \ldots, \mathbf{R}^{n}$, and the spheres $\mathbf{S}^{2}, \mathbf{S}^{3}, \ldots, \mathbf{S}^{n}$ are simply-connected, whereas the circle $\mathbf{S}^{1}$, the cylinder $\mathbf{S}^{1} \times \mathbf{R}$ and the torus $\mathbf{S}^{1} \times \mathbf{S}^{1}$ are multi-connected.

The study of homotopic loops in a manifold $\mathcal{M}$ is a way of detecting holes or handles. Moreover the equivalence classes of homotopic loops can be endowed with a group structure, essentially because loops can be added by joining them end to end. The group of loops is called the first homotopy group at $\mathbf{x}$ or, in the terminology originally introduced by Poincaré, the fundamental group $\pi_{1}(\mathcal{M}, \mathbf{x})$. The fundamental group is independent of the base point: it is a topological invariant of the manifold.

For surfaces, multi-connectedness means that the fundamental group is non trivial: there is at least one loop that cannot be shrunk to a point. But in higher dimensions the problem is more complex because loops, being only one-dimensional structures, are not sufficient to capture all the topological features of the manifolds. The purpose of algebraic topology, extensively developed during the twentieth century, is to generalise the concept of homotopic loops and to define higher homotopy groups. However the fundamental group (the first homotopy group) remains essential.

To define the universal covering space, start with a manifold $\mathcal{M}$ with metric g. Choose a base point $\mathbf{x}$ in $\mathcal{M}$ and consider the differents paths from $\mathbf{x}$ to another point $\mathbf{y}$. Each path belongs to a homotopy class $\gamma$ of loops at $\mathbf{x}$. We construct the universal covering space as the new manifold $(\widetilde{\mathcal{M}}, \tilde{\mathbf{g}})$ such that each point $\tilde{\mathbf{y}}$ of $\widetilde{\mathcal{M}}$ is obtained as a pair $(\mathbf{y}, \gamma)$, y varying over the whole of $\mathcal{M}$ while $\mathbf{x}$ remains fixed and $\gamma$ varies over all homotopy classes. The metric $\tilde{\mathbf{g}}$ is obtained by defining the interval from $\tilde{\mathbf{x}}=(\mathbf{x}, \gamma)$ to a nearby point $\tilde{\mathbf{x}}^{\prime}=\left(\mathbf{x}^{\prime}, \gamma\right)$ in $\widetilde{\mathcal{M}}$ to be 
equal to the interval from $\mathbf{x}$ to $\mathbf{x}^{\prime}$ in $\mathcal{M}$. By construction, $(\widetilde{\mathcal{M}}, \tilde{\mathbf{g}})$ is locally indistinguishable from $(\mathcal{M}, \mathbf{g})$. But its global - namely topological - properties can be quite different. It is clear that, when $\mathcal{M}$ is simply-connected, it is identical to its universal covering space $\widetilde{\mathcal{M}}$. When $\mathcal{M}$ is multi-connected, each point of $\mathcal{M}$ generates an infinite number of points in $\widetilde{\mathcal{M}}$. The universal covering space can thus be thought of as an "unwrapping" of the original manifold.

Consider a point $\mathbf{x}$ and a loop $\gamma$ at $\mathbf{x}$ in $\mathcal{M}$. If $\gamma$ lies entirely in a simply-connected domain of $\mathcal{M},(\mathbf{x}, \gamma)$ generates a single point $\tilde{\mathbf{x}}$ in $\widetilde{\mathcal{M}}$. Otherwise, it generates additional points $\tilde{\mathbf{x}^{\prime}}, \tilde{\mathbf{x}^{\prime \prime}}$, ... which are said to be homologous to $\tilde{\mathbf{x}}$. The displacements $\tilde{\mathbf{x}} \mapsto \tilde{\mathbf{x}}, \tilde{\mathbf{x}} \mapsto \tilde{\mathbf{x}^{\prime \prime}}, \ldots$ are isometries and form the so-called holonomy group $\Gamma$ in $\widetilde{\mathcal{M}}$. This group is discontinuous, i.e., there is a non zero shortest distance between any two homologous points, and the generators of the group (except the identity) have no fixed point. This last property is very restrictive (it excludes for instance the rotations) and allows the classification of all possible holonomy groups.

Equipped with such properties, the holonomy group is said to act freely and discontinuously on $\widetilde{\mathcal{M}}$. The holonomy group is isomorphic to the fundamental group $\pi_{1}(\widetilde{\mathcal{M}})$.

The geometrical properties of a manifold $\mathcal{M}$ within a simply-connected domain are the same as those of its development in the universal covering $\widetilde{\mathcal{M}}$. The largest simply-connected domain containing a given point $\mathbf{x}$ of $\mathcal{M}$, namely the set $\{\mathbf{y} \in \mathcal{M}, d(\tilde{\mathbf{y}}, \tilde{\mathbf{x}}) \leq d(\tilde{\mathbf{y}}, \gamma(\tilde{\mathbf{x}})), \forall \gamma \in \Gamma\}$, is called the fundamental domain $(F D)$.

The $F D$ is always convex and has a finite number of faces (due to the fact that the holonomy group is discrete). These faces are homologous by pairs ; the displacements carrying one face to another are the generators of the holonomy group $\Gamma$.

In two dimensions, the $F D$ is a surface whose boundary is constituted by lines, thus a polygon. In three dimensions, it is a volume bounded by faces, thus a polyhedron.

The configuration formed by the fundamental polyhedron $F D$ and its images $\gamma F D(\gamma \in \Gamma)$ is called a tesselation of $\widetilde{\mathcal{M}}$, each image $\gamma F D$ being a cell of the tesselation.

The $F D$ has two major advantages:

- The fundamental group of a given topological manifold $\mathcal{M}$ is isomorphic to the fundamental group of the $F D$. Routine methods are available to determine the holonomy group of a polyhedron.

- The $F D$ allows one to represent any curve in $\mathcal{M}$, since any portion of a curve lying outside the $F D$ can be carried inside it by appropriate holonomies.

\subsection{TWO-DIMENSIONAL MANIFOLDS}

In addition to pedagogical and illustrative interest, the classification of two-dimensional Riemannian surfaces plays an important role in physics for understanding $(2+1)$-dimensional gravity, a toy model to gain insight into the real world of $(3+1)$-dimensional quantum gravity.

Any Riemannian surface is homeomorphic to a surface admitting a metric with constant curvature $\mathrm{k}$. Thus, any Riemannian surface can be expressed as the quotient $\mathcal{M}=\widetilde{\mathcal{M}} / \Gamma$, where the universal covering space $\widetilde{\mathcal{M}}$ is either (figure 4 ):

- the Euclidean plane $\mathbf{R}^{2}$ if $k=0$

- the sphere $\mathbf{S}^{2}$ if $k>0$

- the hyperbolic plane $\mathbf{H}^{2}$ if $k<0$.

and $\Gamma$ is a discrete subgroup of isometries of $\widetilde{\mathcal{M}}$ without fixed point.

To characterise the quotient spaces we adopt the following abbreviations:

$\mathrm{C}=$ closed, $\mathrm{O}=$ open, $\mathrm{SC}=$ simply-connected, $\mathrm{MC}=$ multi-connected, $\mathrm{OR}=$ orientable, $\mathrm{NOR}=$ non-orientable.

Locally Euclidean surfaces fall into only 5 types: the simply-connected Euclidean plane itself $\mathbf{R}$, the multi-connected cylinder $\mathbf{R} \times \mathbf{S}^{1}$, the Möbius band, the torus $\mathbf{S}^{1} \times \mathbf{S}^{1}$ and the Klein 


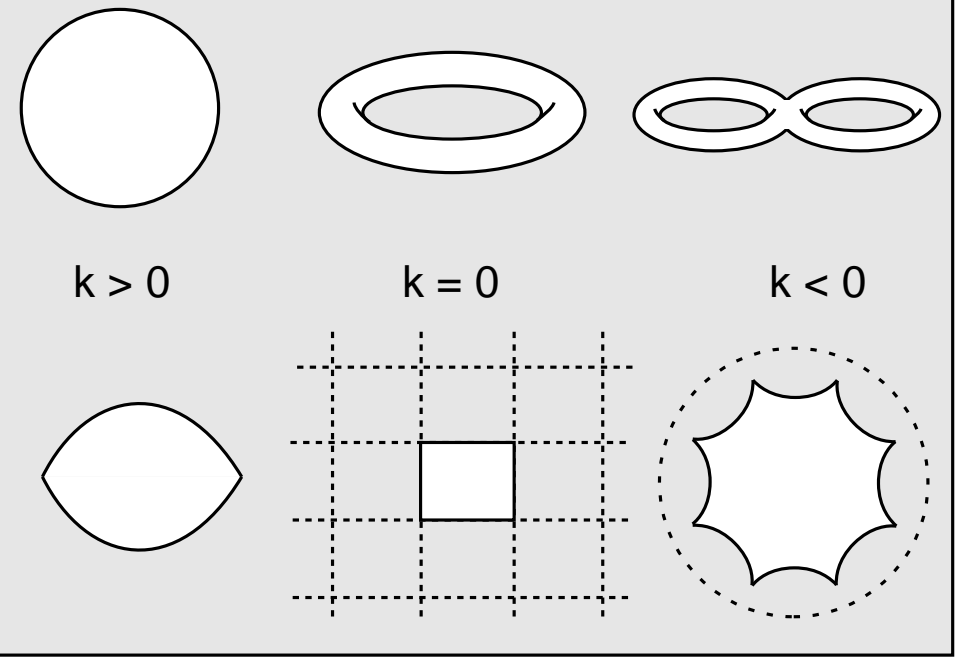

Figure 4. The three kinds of geometries for Riemannian surfaces, as closed surfaces "squeezed" into $\mathbf{R}^{3}$, and as fundamental domains ( $F D$ 's). The covering space for $k>0$ is the sphere $\mathbf{S}^{2}$, and the covering spaces for the other two curvatures are shown schematically.

\begin{tabular}{|c|c|c|c|c|}
\hline Name & $\begin{array}{l}F D \text { and } \\
\text { identifi- } \\
\text { cations }\end{array}$ & Shape & Closed & Orientable \\
\hline cylinder & \begin{tabular}{l|l|l|l|l|l}
$\mathrm{a}$ & & & \\
$\mathrm{b}$ & $\ldots \ldots \ldots$ & \\
$\mathrm{c}$
\end{tabular} & & NO & YES \\
\hline $\begin{array}{l}\text { Möbius } \\
\text { strip }\end{array}$ & & & NO & NO \\
\hline torus & & & YES & YES \\
\hline $\begin{array}{l}\text { Klein } \\
\text { bottle }\end{array}$ & & & YES & NO \\
\hline
\end{tabular}

Figure 5. The four types of multi-connected Euclidean surfaces

bottle. Their characteristics are summarized in figure 5 . The Möbius band and the Klein bottle are not orientable. The torus and the Klein bottle are closed spaces.

There are only two spherical surface forms (this result has been generalized to any constant positive curvature manifold of even dimension): 


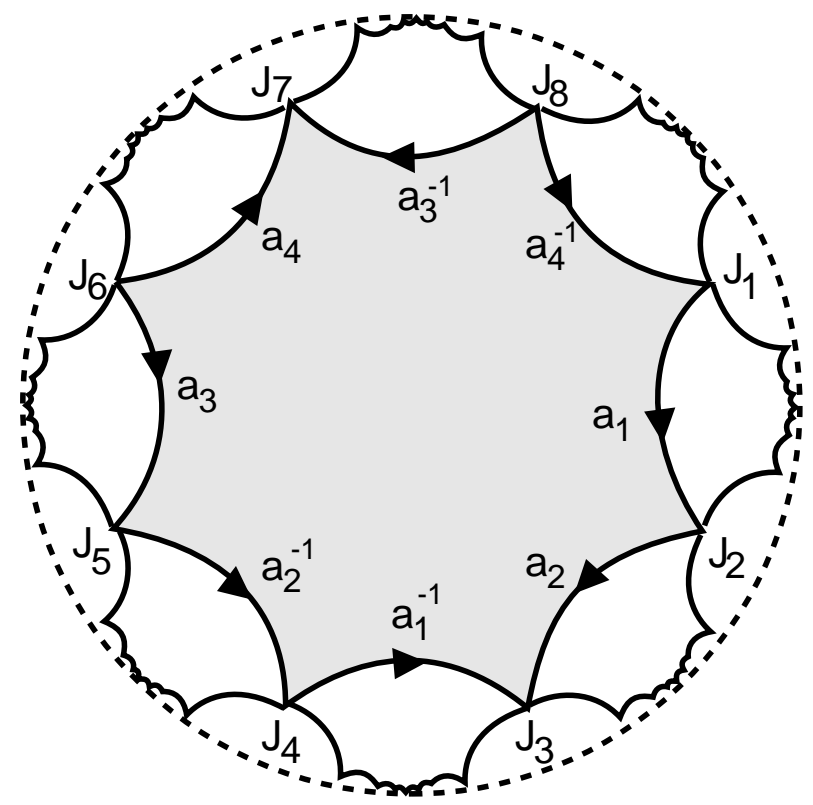

Figure 6. Tesselation of $\mathbf{H}^{2}$ by octagons in the Poincaré representation. The vertices have the coordinates $z\left(J_{k}\right)=0.841 \exp \frac{(3-2 k) \pi i}{8}$

- the sphere $\mathbf{S}^{2}$ itself: C, SC, OR

- the projective plane $\mathbf{P}^{2} \equiv \mathbf{S}^{2} / \mathbf{Z}_{2}$ (also called the elliptic plane): C, MC, NOR.

Whereas the surface of the unit sphere is $4 \pi$, the surface of the unit projective plane is only $2 \pi$, and its diameter, i.e., the distance between the most widely separated points, only $\pi / 2$.

The hyperbolic plane $\mathbf{H}^{2}$, historically known as the Lobachevski space, is difficult to visualize because it cannot be isometrically imbedded in $\mathbf{R}^{3}$. Nevertheless it can be thought of as a surface with a saddle point at every point.

The full isometry group of $\mathbf{H}^{2}$ is $\operatorname{PSL}(2, \mathbf{R}) \equiv S L(2, \mathbf{R}) / \mathbf{Z}_{2}$, where $S L(2, \mathbf{R})$ is the group of real $2 \times 2$ matrices with unit determinant.

The best known example of a compact hyperbolic surface is the 2-torus $T^{2}$. In this case, the $F D$ is a regular octagon with pairs of sides identified. In the Poincaré representation of $\mathbf{H}^{2}$, the $F D$ appears curvilinear. The pavement of the unit disk by homologous octagons (which appear distorted in this representation) corresponds to the tesselation of $\mathbf{H}^{2}$ by regular octagons (figure 6). The famous Dutch artist M.-C. Escher has designed fascinating drawings and prints using such tilings of the hyperbolic plane.

The topological classification of locally hyperbolic surfaces is complete only for the compact $\mathbf{H}^{2} / \Gamma$, which fall into one of the following categories:

- g-torus $T_{g}, g \geq 2$ (connected sum of g simple tori): C, MC, OR

- connected sum of $\mathrm{n}$ projective planes: $\mathrm{C}, \mathrm{MC}, \mathrm{NOR}$

- connected sum of a compact orientable surface $\left(\mathbf{S}^{2}\right.$ or $\left.T_{g}\right)$ and of a projective plane or a Klein bottle: C, MC, NOR

All of these surfaces have a finite area bounded below by $2 \pi$, and a diameter greater than $\cosh ^{-1}(4) \approx 2.06$.

There are an infinite number of non-compact locally hyperbolic surfaces. Clearly, "almost all" Riemannian surfaces are hyperbolic, since:

- Any open surface other than the Euclidean plane, the cylinder and the Möbius band is homeomorphic to a locally hyperbolic surface, for example an hyperbolic plane with or without handles. 
- Any closed surface which is not the sphere, the projective plane, the torus or the Klein bottle is homeomorphic to a locally hyperbolic surface.

\subsection{THREE-DIMENSIONAL MANIFOLDS OF CONSTANT CURVATURE}

Any three-dimensional Riemannian manifold $\mathcal{M}$ admitting at least a 3 -dimensional discrete isometry group $\Gamma$ simply transitive on $\mathcal{M}$ is locally homogeneous and can be written as the quotient $\widetilde{\mathcal{M}} / \Gamma$, where $\overline{\mathcal{M}}$ is the universal covering space of $\mathcal{M}$. Let $G$ be the full group of isometries of $\mathcal{M}$ (containing $\Gamma$ as a discrete subgroup). In the terminology used in the theory of classification of compact three-manifolds, $\mathcal{M}$ is said to admit a geometric structure modelled on $(\widetilde{\mathcal{M}}, G)$.

Thurston has classified the homogeneous three-dimensional geometries into eight distinct types, generally used by mathematicians.

On the other hand, the Bianchi types are defined from the classification of all simplytransitive 3-dimensional Lie groups. Since the isometries of a Riemannian manifold form a Lie group, the Bianchi classification is used by workers in relativity and cosmology for the description of spatially homogeneous spacetimes. The correspondance between the locally homogeneous 3geometries in Thurston's sense and the Bianchi-Kantowski-Sachs classification of homogeneous cosmological models has recently been fully clarified by [63].

Cosmology, however, focuses mainly on locally homogeneous and isotropic spaces, namely those admitting one of the 3 geometries of constant curvature. Any compact 3 -manifold $\mathcal{M}$ with constant curvature $k$ can be expressed as the quotient $\mathcal{M} \equiv \widetilde{\mathcal{M}} / \Gamma$, where the universal covering space $\widetilde{\mathcal{M}}$ is either:

- the 3-sphere $\mathbf{S}^{3}$ if $k>0$

- the Euclidean space $\mathbf{R}^{3}$ if $k=0$

- the hyperbolic 3-space $\mathbf{H}^{3}$ if $k<0$.

and $\Gamma$ is a subgroup of isometries of $\widetilde{\mathcal{M}}$ acting freely and discontinuously.

We give below a schematic description of such spaces.

\subsubsection{Spherical space forms}

Three-manifolds of constant positive curvature have $\mathbf{S}^{3}$, which is compact, as universal covering space. As a consequence they are all compact.

The metric on $\mathbf{S}^{3}$ may be written as

$$
d \sigma^{2}=R^{2}\left\{d \chi^{2}+\sin ^{2} \chi\left(d \theta^{2}+\sin ^{2} \theta d \phi^{2}\right)\right\} .
$$

The volume is $2 \pi^{2} R^{3}$.

The full isometry group of $\mathbf{S}^{3}$ is $S O(4)$. The admissible subgroups $\Gamma$ of $S O(4)$ without fixed point, acting freely and discontinuosly on $\mathbf{S}^{3}$, are:

- the cyclic groups of order $\mathrm{p}, Z_{p}(p \geq 2)$.

- the dihedral groups of order $2 m, D_{m}(m>2)$.

- the polyhedral groups, namely:

- the group $T$ of the tetrahedron (4 vertices, 6 edges, 4 faces), of order 12;

- the group $O$ of the octahedron (6 vertices, 12 edges, 8 faces), of order 24 ;

- the group $I$ of the icosahedron (12 vertices, 30 edges, 20 faces), of order 60 .

All the homogeneous spaces of constant positive curvature are obtained by quotienting $\mathbf{S}^{3}$ with the groups described above. They are in infinite number due to parameters $p$ and $m$. 


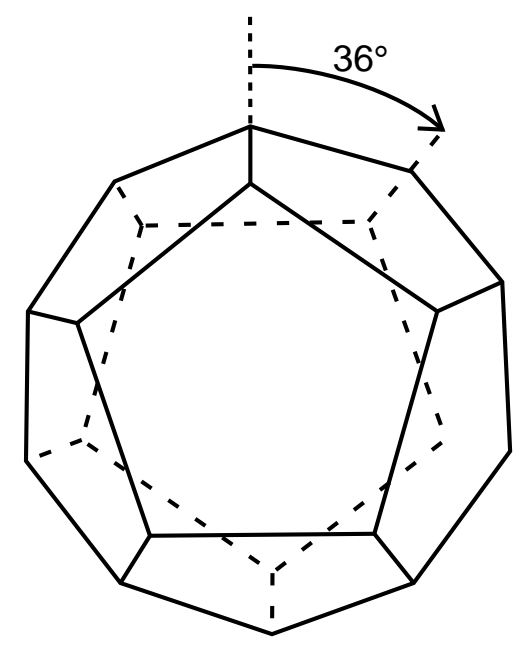

Figure \%. The fundamental domain of the Poincaré spherical space

The volume of $\mathcal{M}=\mathbf{S}^{3} / \Gamma$ is simply

$$
\operatorname{vol}(\mathcal{M})=2 \pi^{2} R^{3} /|\Gamma|
$$

where $|\Gamma|$ is the order of the group $\Gamma$. For topologically complicated spherical 3-manifolds, $|\Gamma|$ becomes large and $\operatorname{vol}(\mathcal{M})$ is small. There is no lower bound since $\Gamma$ can have an arbitrarily large number of elements. Hence

$$
0<\operatorname{vol}(\mathcal{M}) \leq 2 \pi^{2} R^{3}
$$

In contrast, the diameter, i.e., the maximum distance between two points in the space, is bounded below by $\frac{1}{2} \arccos ((1 / \sqrt{3}) \cot (\pi / 5) R \approx 0.326 R$, corresponding to a dodecahedral space.

As well-known examples one can cite

- The projective space $\mathbf{P}^{3}=\mathbf{S}^{3} / \mathbf{Z}_{2}$, obtained by identifying diametrically opposite points on $\mathbf{S}^{3}$. It was used by de Sitter and Lemaitre as the space structure of their cosmological models.

- The lens spaces $\mathbf{S}^{3} / \mathbf{Z}_{p}$. The simplest one, $\mathbf{S}^{3} / \mathbf{Z}_{3}$, divides $\mathbf{S}^{3}$ into 6 fundamental cells, each having a lens form.

- The Poincaré dodecahedral space is an example of $\mathbf{S}^{3} / I$. The fundamental polyhedron is a regular dodecahedron of which the faces are pentagons. The compact space is obtained by identifying the opposite faces after rotating by $1 / 10^{t h}$ turn in the clockwise direction around the axis orthogonal to the face (figure 7). This configuration involves 120 successive operations and gives already some idea of the extreme complication of such multi-connected topologies.

\subsubsection{Euclidean space forms}

The line element for the universal covering space $\mathbf{R}^{3}$ may be written as:

$$
d \sigma^{2}=R^{2}\left\{d \chi^{2}+\chi^{2}\left(d \theta^{2}+\sin ^{2} \theta d \phi^{2}\right)\right\}
$$

Its full isometry group is $G=I S O(3) \equiv \mathbf{R}^{3} \times S O(3)$, and the generators of the possible holonomy groups $\Gamma$ (i.e., discrete subgroups without fixed point) include the identity, the translations, the glide reflections and the helicoidal motions occurring in various combinations. They 

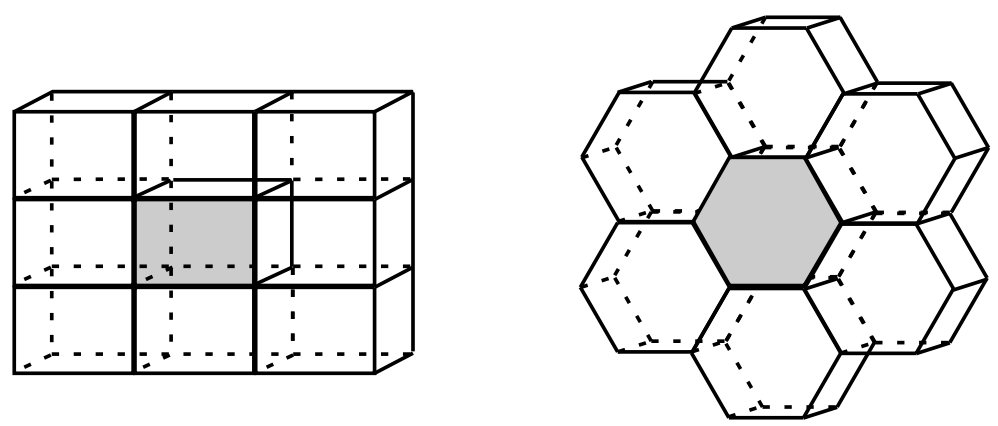

Figure 8. Tesselation of $\mathbf{R}^{3}$ by parallelepipeds or hexagonal cells

generate 18 distinct types of locally Euclidean spaces. The 17 multi-connected space forms are in correspondance with the 17 crystallographic groups discovered more than a century ago by Feodoroff. Eight forms are open (non compact), ten are closed (compact).

The compact models can be better visualised by identifying appropriate faces of fundamental polyhedra. Six of them are orientable. The fundamental polyhedron can be a parallelepiped or a hexagonal prism, with various possible possible identifications. The 3 -torus is the simplest one. Their volume is arbitrary, since there is no rigidity theorem linking the curvature radius to the topological lengths. Until 1985 [23, 24, 26], such spaces were nearly the only ones to be explored for discussion of cosmic topology.

\subsubsection{Hyperbolic space forms}

Locally hyperbolic manifolds are less well understood than the other homogeneous spaces. However, according to the pioneering work of Thurston, "almost all" 3 -manifolds can be endowed with a hyperbolic structure.

The induced metric on $\mathbf{H}^{3}$ may be written as

$$
d \sigma^{2}=R^{2}\left\{d \chi^{2}+\sinh ^{2} \chi\left(d \theta^{2}+\sin ^{2} \theta d \phi^{2}\right)\right\}
$$

The volume of $\mathbf{H}^{3}$ is infinite. Its group of isometries is isomorphic to $\operatorname{PSL}(2, \mathbf{C})$, namely the group of fractional linear transformations acting on the complex plane. Finite subgroups are discussed in [3].

In hyperbolic geometry there is an essential difference between the 2-dimensional case and higher dimensions. A surface of genus $g \geq 2$ supports uncountably many non equivalent hyperbolic metrics. But for $n \geq 3$, a connected oriented $n$-manifold supports at most one hyperbolic metric. More precisely, the rigidity theorem proves that (for a fixed value of $R$ ) if two hyperbolic manifolds, with dimension $n \geq 3$, have isomorphic fundamental groups, they are necessarily isometric to each other. It follows that, for $n \geq 3$, the volume of a manifold and the lengths of its closed geodesics are topological invariants. This suggested the idea of using the volumes to classify the topologies, which could have seemed, at a first glance, contradictory with the very purpose of topology.

Each type of topology is characterized by some lengths. For compact locally Euclidean spaces, the fundamental domain may possess arbitrary volume, but no more than eight faces. In the spherical case, the volume of $\mathbf{S}^{3} / \Gamma$ is finite and is that of $\mathbf{S}^{3}$, the maximum possible value, divided by a whole number. By contrast, it is possible to tesselate $\mathbf{H}^{3}$ with polyhedra having an arbitrarily large number of faces. In the three-dimensional hyperbolic case, the possible values for the volume of the $F D$ are bounded from below. In other words, there exist hyperbolic 3manifolds with volumes arbitrarily close to a minimal volume for all hyperbolic 3-manifolds. 

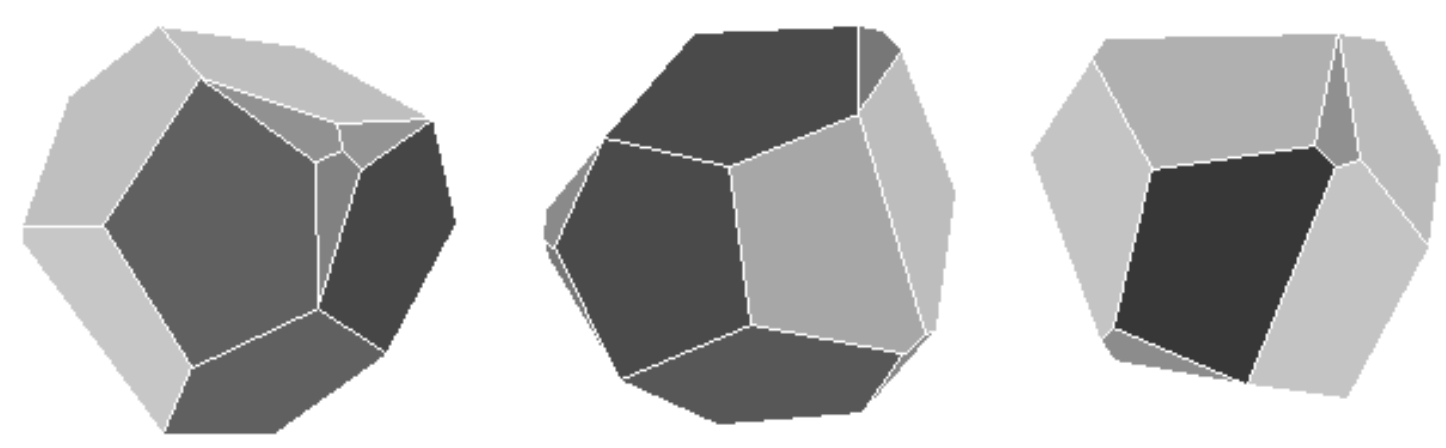

Figure 9. Three views of the fundamental domain of the Weeks manifold

Particular interest has been taken by various authors in computing the volumes of compact hyperbolic manifolds. Each topology has a specific volume measured in curvature radius units. The absolute lower bound for the volume of CHMs is given by $V_{\min }=0.16668$ [35]. However, it may have little effect on cosmological applications. The reason is that the true lower bound is expected to be 0.942707 , corresponding to the smallest CHM that is presently known [85, 58]. The new $V_{\min }$ bound represents an improvement in the techniques of the proof, not an increase in the expected size of the smallest hyperbolic manifold.

In view of cosmological observational effects, the smaller the value of vol $_{\min }$, the more interesting the corresponding manifold for cosmology. Thus the Weeks space has been specially studied, by [25] and more recently by [47]. Its $F D$ is a polyhedron with 26 vertices and 18 faces, of which 12 are pentagons and 6 are quadrilaterals. Its outer structure is represented in figure (9), the Klein coordinates of the vertices and the 18 matrix representations of the generators of the holonomy group are given in [47].

The Weeks manifold leaves room for many topological lens effects, since the volume of the observable universe is about 200 times larger than the volume of Weeks space for $\Omega_{0}=0.3$. Indeed, many CHMs have geodesics shorter than the curvature radius, leaving room to fit a great many copies of a fundamental polyhedron within the horizon radius, even for manifolds of volume $\sim 10$. The publicly available program SNAPPEA (Weeks) is specially useful to unveil the rich structure of CHMs. Several millions of CHMs with volume less than 10 could be calculated. Table 1 summarizes a sample of the results $\left(r_{-}\right.$is the radius of the largest sphere in the covering space which can be inscribed in the fundamental polyhedron, $r_{+}$is the radius of the smallest sphere in the covering space in which the fundamental polyhedron can be inscribed, $l_{\text {min }} \equiv 2 r_{\text {inj }}$ is the length of the shortest geodesic; see Fig. 10).

\section{Topology and Cosmology}

It is presently believed that our Universe is correctly described by a perturbed FriedmannLemaitre (FL) model. That is, homogeneous and isotropic solutions of Einstein's equations are used, of which the spatial sections have constant curvature. The observational fact that the Universe is, in an exact sense, inhomogeneous and anisotropic, is modelled by perturbing these solutions. Beside the usual "big-bang" solutions, the FL models also include the de Sitter solution, as well as those incorporating a cosmological constant, or a non standard equation of state. From a spatial point of view, the FL models fall into 3 general classes, according to the sign of their spatial curvature $k=-1,0$, or +1 . The spacetime manifold is described by the Robertson-Walker metric

$$
d s^{2}=c^{2} d t^{2}-R^{2}(t) d \sigma^{2}
$$


TABLE 1. Small CHMs from SnapPea

\begin{tabular}{ccccc}
\hline Name & Volume & $r_{-}$ & $r_{+}$ & $l_{\min }$ \\
\hline WMF & 0.9427 & 0.5192 & 0.7525 & 0.5846 \\
Thurston & 0.9814 & 0.5354 & 0.7485 & 0.5780 \\
s556(-1,1) & 1.0156 & 0.5276 & 0.7518 & 0.8317 \\
$\mathrm{~m} 006(-1,2)$ & 1.2637 & 0.5502 & 0.8373 & 0.5750 \\
$\mathrm{~m} 188(-1,1)$ & 1.2845 & 0.5335 & 0.9002 & 0.4804 \\
$\mathrm{v} 2030(1,1)$ & 1.3956 & 0.5483 & 1.0361 & 0.3662 \\
$\mathrm{~m} 015(4,1)$ & 1.4124 & 0.5584 & 0.8941 & 0.7942 \\
$\mathrm{~s} 718(1,1)$ & 2.2726 & 0.6837 & 0.9692 & 0.3392 \\
$\mathrm{~m} 120(-6,1)$ & 3.1411 & 0.7269 & 1.2252 & 0.3140 \\
$\mathrm{~s} 654(-3,1)$ & 4.0855 & 0.7834 & 1.1918 & 0.3118 \\
$\mathrm{v} 2833(2,3)$ & 5.0629 & 0.7967 & 1.3322 & 0.4860 \\
$\mathrm{v} 3509(4,3)$ & 6.2392 & 0.9050 & 1.3013 & 0.3458
\end{tabular}

where

$$
d \sigma^{2}=d \chi^{2}+S_{k}^{2}(\chi)\left(d \theta^{2}+\sin ^{2} \theta d \phi^{2}\right)
$$

is the metric of a 3 -dimensional homogeneous manifold, flat $[k=0$, see eq. (4)] or with curvature $[k= \pm 1$, see eqs $(1),(5)]$. We have defined the function

$$
\begin{cases}S_{k}(\chi)=\sinh (\chi) & \text { if } k=-1 \\ S_{k}(\chi)=\chi & \text { if } k=0 \\ S_{k}(\chi)=\sin (\chi) & \text { if } k=1\end{cases}
$$

and $R(t)$ is the scale factor, chosen equal to the spatial curvature radius for non flat models, so that $k / R^{2}$ is the spatial curvature.

The most usually considered cosmological models are those with simply-connected space. We will refer generically to them as the Simply-Connected Models, hereafter SCM's. However, the assumption of simple-connectedness is arbitrary and can be dropped out. The multi-connected cosmological models (hereafter MCM's) are those having multi-connected spatial sections. To any MCM is associated a unique SCM sharing exactly the same kinematics and dynamics. The universal covering (UC) of the spatial sections of an MCM is the spatial section of the corresponding SCM $\left(\mathbf{R}^{3}, \mathbf{H}^{3}\right.$ or $\mathbf{S}^{3}$ for FL models $)$ at the cosmological time defining the spatial section. In particular, the scale factors $R(t)$ are exactly identical. In fact, most characteristics of the Friedmann-Lemaître models are preserved when we turn to the MCM's, and this is a precious guide for their study.

The values of the metric parameters [the density parameter $\Omega_{0}$ and the dimensionless cosmological constant, $\lambda_{0} \equiv c^{2} \Lambda /\left(3 H_{0}^{2}\right)$; in the notation of [61] $\lambda_{0} \equiv \Omega_{\Lambda}$ ] are not known accurately enough to decide the sign of the curvature. In fact, it is difficult to see how dynamical measurements can distinguish between $\Omega_{0}+\lambda_{0}=1$ and $\Omega_{0}+\lambda_{0}=1 \pm \delta$ where $0<\delta \ll 1$. On the contrary, significant detection of non-trivial topology would dictate the sign of the spatial curvature, since the structures of the holonomy groups are completely different for the cases $k=1,0,-1$. Moreover, it is worthwhile to note that the multi-connectedness of space would provide precise constraints on the metric parameters independently of the cosmic distance ladder $[71]$.

Thus, beyond its own specific interest, multi-connectedness would offer a very efficient tool of investigation in other aspects of observational cosmology.

In SCM's, the only scale in comoving space which is not fixed is the curvature radius, normally defined as the present value of the scale factor $R\left(t_{0}\right)=R_{C}$ (there is no scale at all in the flat 
case). Thus $R_{C}$ is the natural length unit in comoving space for the UC of an MCM. The Friedmann equations imply the relation

$$
\Omega_{0}+\lambda_{0}-1=\frac{k c^{2}}{H_{0}^{2} R_{C}^{2}}
$$

The value of $R_{C}$ remains a matter of considerable debate. The only cosmological length to which we have a direct observational access is the Hubble length

$$
L_{\text {Hubble }}=\mathrm{cH}_{0}^{-1}=3000 \mathrm{~h}^{-1} \mathrm{Mpc} .
$$

If we define $f=\sqrt{|\Omega+\lambda-1|}$, we have for a non-flat universe (for a flat universe, the value of $R_{C}$ remains arbitrary):

$$
R_{C}=L_{\text {Hubble }} f^{-1}=3000(f h)^{-1} \mathrm{Mpc} .
$$

In practice, the Hubble length is of the same order of magnitude as $R_{H}$, the particle horizon, which defines the observable universe as a sphere in the UC. This is just slightly larger than the radius to the last scattering surface, which is the present limit to observations.

Another natural cosmological length is associated with the cosmological constant:

$$
L_{\Lambda}=\sqrt{1 / \Lambda}=\sqrt{\left(3 H_{0}^{2} \lambda_{0} / c^{2}\right)^{-1}}=1730 / \sqrt{\lambda_{0}} \mathrm{~h}^{-1} \mathrm{Mpc} .
$$

The concept of horizon keeps its exact validity in the MCM's, but must be applied to the universal covering space: an image is potentially visible iff its (comoving) distance is smaller than $R_{H}$ in the universal covering.

In an MCM, additional spatial scales are associated with the topology, those of the fundamental polyhedron. The geometry suggests to compare them with $R_{C}$ but it is often more convenient, for observations, to compare them to $R_{H}$, or to evaluate them in $\mathrm{Mpc} \mathrm{or} \mathrm{h}^{-1} \mathrm{Mpc}$.

Figure 10 and its caption give the basic definitions. Observable effects linked to the multiconnectedness will only occur if these scales are smaller than the size of the observable universe, i.e., the horizon radius.

Dropping the hypothesis that real space is simply-connected has various implications. If at least one of the topological scales is smaller than the horizon, then this will, in principle, be observable: multiple images of the same object or radiation emitting region will exist. The smaller the fundamental domain, the easier it is to observe the multiple topological imaging. It has recently been calculated [47], for a given catalog of observable cosmic sources (discrete or diffuse) with a given depth in redshift, the approximate number of topological images in locally hyperbolic and locally elliptic spaces as a function of the cosmological paramaters $\Omega_{0}$ and $\lambda_{0}$. How do the present observational data constrain the possible multi-connectedness of the universe? And, more generally, what kinds of tests are conceivable ? The following section deals with these matters.

\section{Observational methods, candidates and constraints}

\section{1. "TOPOLOGY" FOR THE OBSERVER}

The simplest observational point of view for approaching cosmological topology is to consider the observable sphere, in comoving coordinates, in the covering space.

For brevity, an abuse of language is often adopted where a "topology" is considered to mean a specific 3-manifold, expressed with some or all of the quantitative parameters in physical units with a definite orientation, in some standard astronomical coordinate system. Formally, this can be expressed as the set

$$
\left\{\widetilde{M} \equiv \kappa_{0} \equiv \Omega_{0}+\lambda_{0}-1, \Omega_{0}, \Gamma, \delta \widetilde{M}, \delta \Omega_{0}, \delta \Gamma\right\}
$$




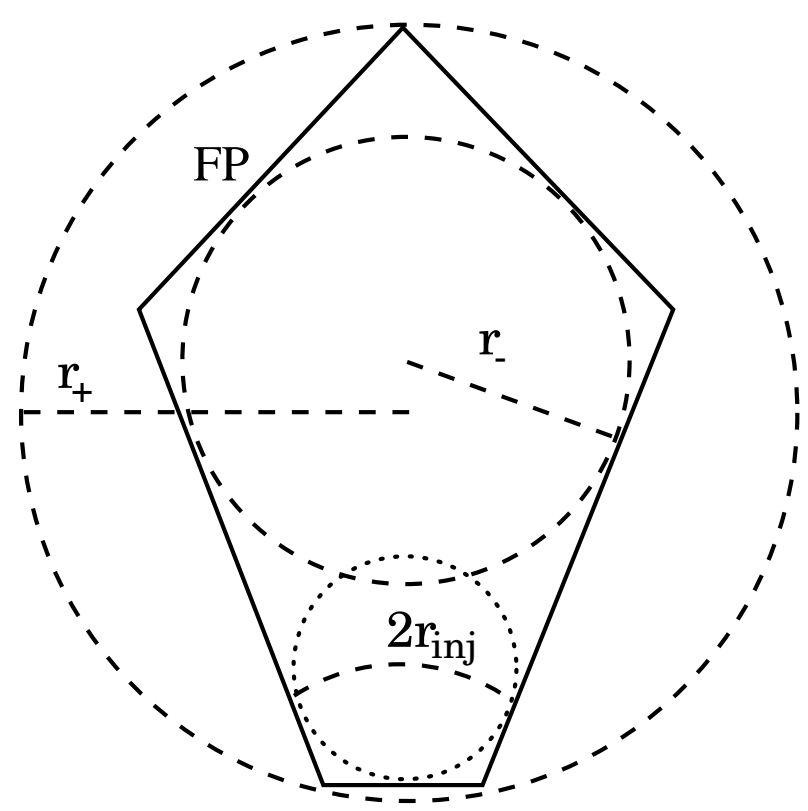

Figure 10. Schematic diagram showing key parameters representing the size of a small universe. The terminology is that of [9]. The in-radius $r_{-}$and the out-radius $r_{+}$are characterised by the largest sphere inscribable in the fundamental domain $(F D)$ and the smallest sphere circumscribable around the $F D$ respectively. The injectivity radius $r_{i n j}$ is half of the smallest closed (spatial) geodesic from one topological image to another. The particular geometry shown does not necessarily correspond to a projection of a slice through a 3-manifold known to exist, it is for demonstration only.

where $\widetilde{M}$ is the covering space, which both determines and is determined uniquely by the curvature under the assumption of constant curvature; $\Omega_{0}$ is the present-day value of the density parameter, required (along with $\lambda_{0}$, which satisfies $\lambda_{0}=\kappa_{0}-\Omega_{0}+1$ ) in order to relate cosmological redshift $z$ and distance; $\Gamma=\left\{g_{i}\right\}$ is the holonomy group of isometric linear transformations within the covering space which map topological images onto one another, (e.g. combinations of translations, rotations and reflections, if $\widetilde{M}=\mathbf{R}^{3}$ ); and $\delta$ is used to indicate (in abbreviated form) that the observational uncertainties in all the quantitative parameters need to be determined.

An equivalent, more physical, way of thinking of this set is in terms of the $F D$, or fundamental polyhedron, which we label $P$, i.e.

$$
\left\{P \equiv \widetilde{M} / \Gamma, \Omega_{0}, \Gamma, \delta P, \delta \Omega_{0}, \delta \Gamma\right\}
$$

where $P$ is just the polyhedron shape, orientation and our location within it (all represented quantitatively in physical units and coordinates), without the generators, and $\Gamma$ lists the generators (also in physical units and coordinates).

The values of $\kappa_{0}$ of most observational interest are $\kappa_{0} \leq 0$. Fig. 11 puts into perspective the length scales corresponding to the range in $\kappa_{0}$ and $\Omega_{0}$ [expressed as the pair $\left(\Omega_{0}, \lambda_{0}\right)$ ] considered consistent with present observations.

In the cases of zero curvature, no geometric constraint exists on how many copies of $P$ could exist within the observable sphere. In the hyperbolic case, values of $2 r_{\text {inj }} / R_{C} \lesssim 0.5$ are common for manifolds in the census available with SnAPPEA (e.g. Table 1), i.e. $2 r_{\text {inj }} \lesssim 1700 \mathrm{~h}^{-1} \mathrm{Mpc}$ for the hyperbolic covering space $\widetilde{M}$ shown. So, the constraint of the rigidity theorem in the hyperbolic case does not prevent the possibility of multiple topological images at redshifts considerably smaller than $z=3$. 
TABLE 2. Summary of the most recent methods and observational results. See text ( $(55)$ for details. Author abbreviations are by authors' surnames' initials and year.

\begin{tabular}{|c|c|c|c|c|}
\hline $\begin{array}{l}\text { (1) Meth } \\
\text { 3D: } \\
\text { clus opt } \\
\text { clus Xray } \\
\text { QSO's }\end{array}$ & $\begin{array}{l}\text { ds: } \\
\text { cosmic crystallog. } \\
\text { brightest cluster } \\
\text { local isom. search }\end{array}$ & $\begin{array}{l}\text { LLL } 96 \\
\text { RE } 97\end{array}$ & \multicolumn{2}{|l|}{ R 96} \\
\hline $\begin{array}{l}\text { 2D: } \\
\text { CMB }\end{array}$ & $\begin{array}{l}\text { ID'd circles } \\
C_{l}-\text { cutoff } \\
\text { correlation fn }\end{array}$ & & & $\begin{array}{c}\text { CSS96/98 } \\
\text { many } \\
\text { BPS98 }\end{array}$ \\
\hline Ideal objec & $\begin{array}{c}\text { no Evoln } \\
\text { zero pec velocity } \\
\text { isotropic emitter } \\
\text { seen to large } z \\
\text { seen over large vol } \\
\text { seen to }\left|b^{I I}\right| \ll 20^{\circ}\end{array}$ & $\begin{array}{c}\text { monotonic } \mathrm{E} \\
\text { prob. small } \\
\mathrm{Y}(\text { nearly }) \\
\mathrm{Y}\left(\kappa_{0}<0\right), \mathrm{N}(\text { o.w. }) \\
\mathrm{N} \\
\mathrm{N}\end{array}$ & $\begin{array}{c}\text { strong E } \\
\text { prob. small } \\
\text { N } \\
\text { Y } \\
\text { Y } \\
\text { N }\end{array}$ & $\begin{array}{c}\text { weak E? } \\
\text { N } \\
\text { Y/N } \\
\text { Y } \\
\text { Y (sph shell) } \\
\text { N }\end{array}$ \\
\hline Assumptio & s on $\kappa_{0},\left\{g_{i}\right\}$, ideal & one: & $\kappa_{0}$ (use range) & $\begin{array}{c}\text { circles: none } \\
C_{l} \text { : all }\end{array}$ \\
\hline
\end{tabular}

(2) Constraints:

CC: $2 r_{+} \gtrsim R_{H} / 20$
BC: $2 r_{+} \gtrsim R_{H} / 10$

For the following special cases, but really testing perturbation spectrum assumptions:

$\kappa_{0}=0$, if $\theta\left(g_{i}, g_{j}\right)=90 i^{\circ}$ or $60 i^{\circ}, i \in Z$ then

$\kappa_{0}<0$, if $\Gamma=m 004(-5,1)$ or $\Gamma=v 3543(2,3)$ then $\quad \begin{gathered}\left(2 r_{\text {inj }} \gtrsim R_{H} / 2\right) \\ \left(2 r_{\text {inj }} \gtrsim 2 R_{H}\right)\end{gathered}$

(2) Specific candidates:

\begin{tabular}{|ccc} 
serendipitous & $2 \sigma$ implicit & "preferable \\
$\kappa_{0}=0$ & $\kappa_{0}<0 ?$ & to SCDM" \\
$\widetilde{M} / \Gamma=T^{2} \times \mathbf{R}$ & [non-orientable] & $\mathrm{v} 3543(2,3)$ \\
$2 r_{\text {inj }}\left(\Omega_{0}\right)=$ & & $r_{\text {inj }}=0.95 R_{H}$ \\
$965 \pm 5 h^{-1} \mathrm{Mpc}(1)$ & & \\
$1190 \pm 10 h^{-1} \mathrm{Mpc}(0.2)$ & & \\
RE97, RB99 & R96 & BPS98 \\
\hline
\end{tabular}

\subsection{EARLY WORK: $\lesssim 100 \mathrm{~h}^{-1} \mathrm{Mpc}$}

Sections 11 and 12 of [45] review many different efforts to detect or constrain the value of $r_{\text {inj }}$.

Note that the different parameters expressing the size of the Universe, $r_{\text {inj }}, r_{-}$and $r_{+}$, in particular, (Fig. 10) have not always been carefully distinguished. For example, the fact that we live in the disk of a spiral galaxy, in which dust obscures most extragalactic observations, implies that many lower limits estimated for the "size" of $P$ are in fact lower limits on $r_{+}$, but 

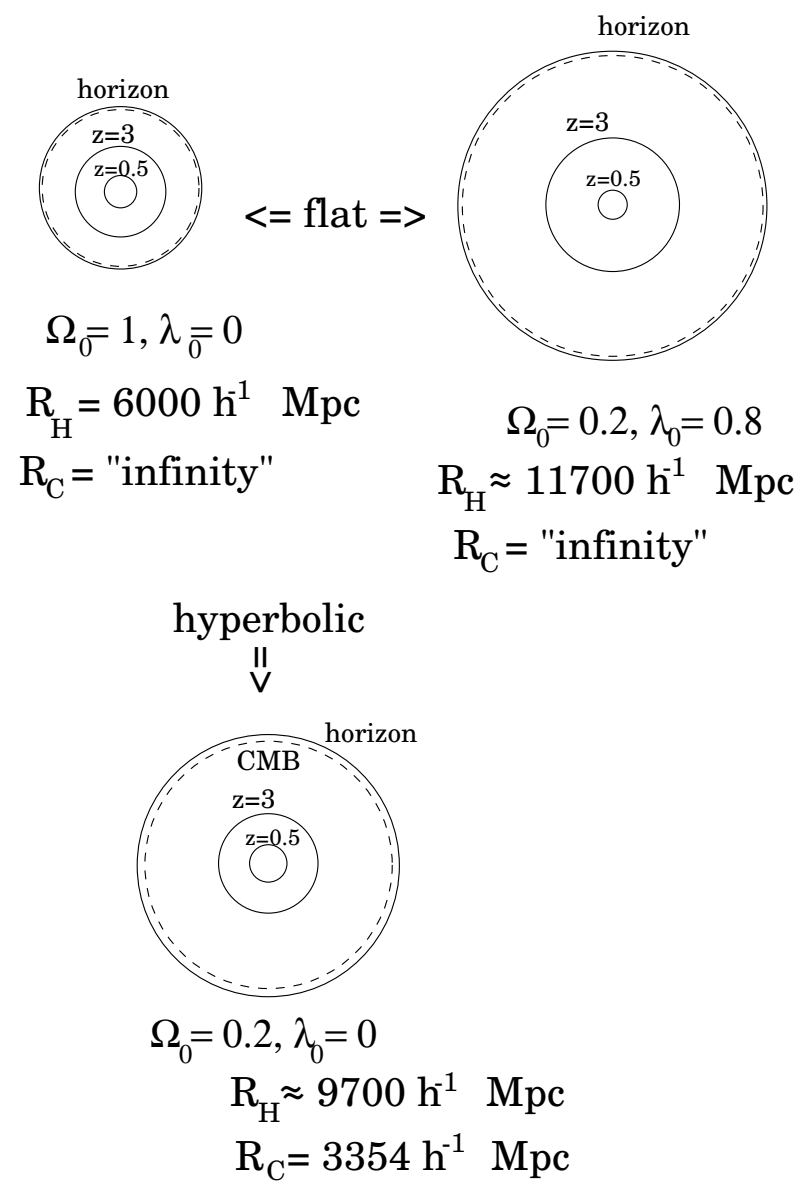

Figure 11. Relative sizes of the observable Universe, depending on different options for the metric parameters $\Omega_{0}, \lambda_{0}$, shown in comoving coordinates on a radially linear (proper distance) scale. The horizon radius $R_{H}$ is defined by the age of the Universe and the curvature radius is $R_{C} \equiv c / H_{0}\left(\left|1-\Omega_{0}-\lambda_{0}\right|\right)^{-1 / 2}$. The inner circles for each choice of metric indicate scales to which we can see: X-ray emission from the richest clusters of galaxies $(z \sim 0.5)$, large numbers of quasars $(z \sim 3)$, the cosmic microwave background $(z \sim 1100$, dashed circle). In the flat models, the tangential distance scale is constant and the same as the radial scales; in the hyperbolic model, the tangential distance scale increases (more Gpc per $\mathrm{mm}$ on the page) as a function of increasing radius, i.e. it is $\left[\sinh \left(r / R_{C}\right)\right] /\left(r / R_{C}\right)$ times larger than a constant scale, e.g. the horizon circumference is $3.1\left(2 \pi R_{H}\right)$.

not on $r_{\text {inj. }}$.

However, the constraints at $r_{\text {inj }} \lesssim 100 \mathrm{~h}^{-1} \mathrm{Mpc}$ are strong. For example, close images of the Coma cluster do not exist, except if hidden by the galactic plane [37], and large scale structure (walls, filaments, voids) clearly exists and intuitively it is difficult to see how the Universe could be much smaller. So $2 r_{+} \gtrsim R_{H} / 100$ is a fair estimate.

\subsection{SINCE 1993: $\gg 100 \mathrm{~h}^{-1} \mathrm{Mpc}$}

At larger scales, with increasing amounts of data available from better telescopes, observational work on cosmological topology has increased rapidly in the last few years. Some methods test for specific candidates or families of 3-manifold candidates (e.g. [5]), but several new generic methods have been developed which avoid having to assume a specific manifold to start off with $[46,65,70,10]$.

The principle common to all methods is that multiple topological images of "objects" should exist, if $r_{\mathrm{inj}}<R_{H}$, where in the case of the cosmological microwave background (CMB), regions 


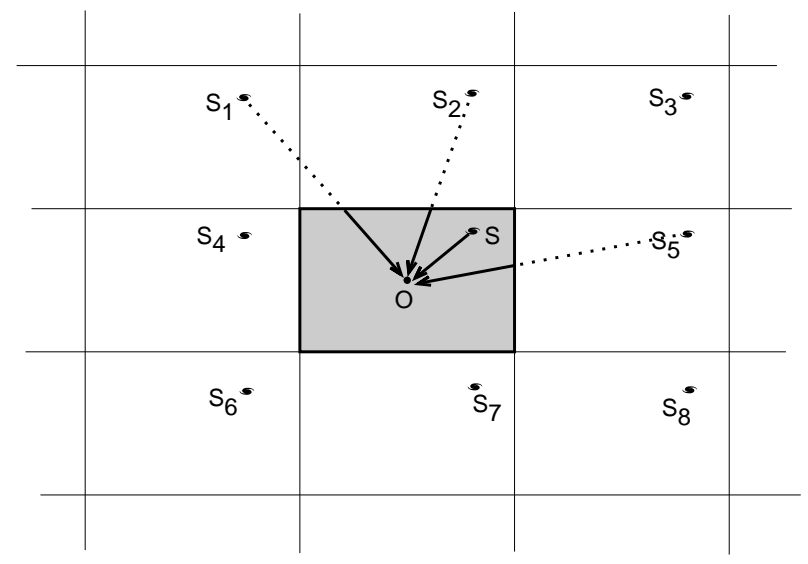

Figure 12. Multiplication of images in the universal covering space of a toroidal universe

of space whose black-body temperature can be measured are considered as objects, though they are not astrophysical objects in the ordinary sense. This is illustrated for the flat torus model in Fig. 12.

What varies between the methods depends on many factors. The intrinsic properties of the "objects" (or the lack of knowledge of their properties), their intrinsic distribution throughout the observed time cone and relative to the galactic plane, and their visibility, make finding "spatially well distributed, standard candles" difficult.

Specifically, an ideal "object" (whether a collapsed object, a "configuration" of objects, or a patch of plasma in the CMB) should:

(i) not evolve with lookback time

(ii) have zero three-dimensional peculiar velocity

(iii) emit isotropically

(iv) be seen to large redshifts (distances)

(v) be seen over a large total volume (range in redshifts)

(vi) not be obscurable by dust in the galactic plane, nor by high latitude dust (e.g. Ophiuchus, Orion)

Objects which are probably the best standard candles, i.e. clusters of galaxies detected by their hot gas in X-rays, are mostly only seen to very small fractions of $R_{H}$ (though future observations may improve this, particularly if the curvature $\kappa_{0}$ is negative enough). Objects seen to large fractions of $R_{H}$, such as quasars, are probably the worst standard candles.

This is, of course, the basic difficulty in observational cosmology, shared by the attempts to measure the metric parameters $\Omega_{0}, \lambda_{0}$ and $H_{0}$.

The different methods, their advantages and disadvantages, the claimed constraints so far, and, moreover, suggested candidates for the 3-manifold [for "the topology" in the sense defined by eq. (10)] are listed in Table 2. At least one of the suggested candidates makes predictions which would be refutable with a modest size observing programme on major telescopes.

\subsubsection{Methods: three-dimensional}

For a full review of three-dimensional observational methods, i.e. those using astrophysical objects distributed throughout the three-dimensional observable sphere of the covering space, see [69]. Only a brief description is provided here.

(i) The principle behind the method of [46], "cosmic crystallography", is that for good standard candles, multiples of the vectors corresponding to the generators, should cause very sharp peaks in a histogram of the separations of pairs of objects (non-normalised two-point correlation 


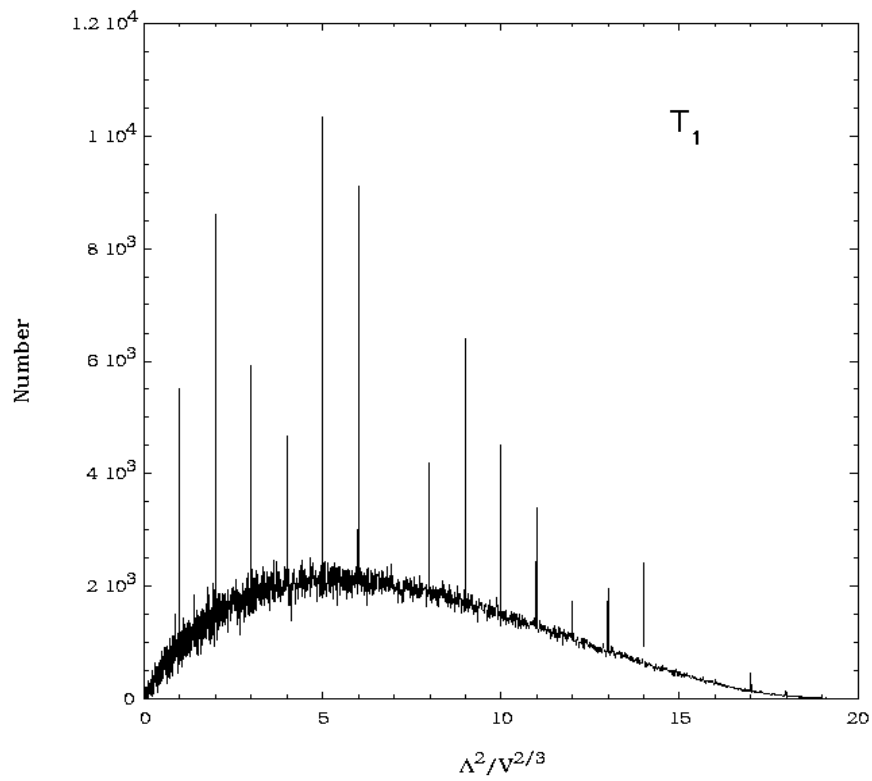

Figure 13. Histogram of pair separations for a computer generated Einstein-de Sitter universe. Fifty galaxies up are randomly distributed in a cubic fundamental domain of size $2500 \mathrm{Mpc}$. Topological images are calculated up to a redshift 4 . The amplitude and relative positions of spikes reveal the holonomies of space and the topological type.

function), see Fig. 13. Rich clusters of galaxies, particularly if selected in X-ray surveys, should be fairly good standard candles at redshifts smaller than their formation redshifts, simply because they are dominated by hot gas in kinetic equilibrium which doesn't have time within the age of the Universe to evolve much except to grow by accretion and become hotter and brighter. In addition clusters, again preferably seen by the X-ray gas, should be relatively isotropic emitters (compared to, say, quasars).

Lehoucq et al. [46] performed their simulations in Euclidean spaces only and assumed that the other curvature cases gave similar results. The efficiency of the method has also been discussed by [28] when the size of the physical space is comparable to the horizon size.

Lehoucq et al. [47] have next analysed the applicability of the method to elliptic and hyperbolic spaces. In the first case there may be topological signal in the histogram. However, small elliptical spaces are not experimentally favored by the present estimates of the age of the universe. In the hyperbolic case, no peaks in the pair 3D separations histogram were observed, due to the fact that the number of copies of the fundamental domain in the observable covering space is low and that the points are not moved the same distances by the holonomies of space. The lack of peaks in the hyperbolic case has simultaneously been noticed by [29] and [36].

Both [29] and [83] have devised improvements on the crystallographic method aimed to extract all the topological signal the histograms, either by subtracting out the smooth (nontopological) signal or by considering differential histograms.

(ii) In the absence of deep, wide angle surveys for what are the best known standard candles (galaxy clusters), other approaches are needed.

Another use of standard candles (cf. [37]) with (most likely) monotonic evolutionary properties, i.e. clusters seen in X-rays, is that if the highest redshift cluster in a systematic, complete, all-sky survey is at a high redshift, then no topological images of it can exist at lower redshifts, since they would have to be brighter than the high redshift cluster, which in that case would not be the brightest [70]. 
(iii) At larger scales, the objects most readily available are quasars. These are not good standard candles, and either exist (as quasars) only for small fractions of the age of the Universe, or recur as bursts several times. In addition, they would be seen (except in special cases) from different angles, and so appear as another form of active galactic nucleus (AGN) much fainter, and not yet catalogued.

The way around this problem [65] is to look for, in a large enough catalogue, the rare cases in which the evolution (and orientation) of the quasars happens to be ideal for an Earth-based observer, and multiple topological images are seen of several quasars in two topological images of a "small region", i.e. of a region of a few $100 \mathrm{~h}^{-1} \mathrm{Mpc}$ in size. The method is then to search (in 3-D, using redshifts for distance estimation) for all possible isometries between configurations of quasars in such "small regions". There is not much time for the relative positions of quasars to change much within a configuration, so in a sense, the configurations correspond to pseudoobjects of size $100 \mathrm{~h}^{-1} \mathrm{Mpc}$ which, in a small number of cases, effectively do not evolve.

Given contamination by chance isometries of configurations unrelated to topology [65], theoretical work (analytical and/or simulations) is required to find the statistically optimal implementations of this method. For example, although weakening the isometry criteria (e.g. matching quadruplets instead of quintuplets) would increase the number of non-topological (noise) isometries, it might increase the number of topological (signal) matches by a greater factor.

(iv) A method not yet applied directly, though implicit in some sense in the above methods, is to consider units of "large scale structure" (walls, voids, etc. on scales of $50-150 \mathrm{~h}^{-1} \mathrm{Mpc}$ ) as objects. Although individual galaxies and quasars will be difficult to identify at different epochs, it is expected that they should trace out thin structures which should not move much over the time scales involved [37]. One approach to analysing the data to be taken by the X-ray satellite $\mathrm{XMM}$ is to use the representations of large scale structure by the 2-dimensional topology of contours of constant density as a characteristic to identify potential multiple topological images of these structures. In other words, the (2-D) topology of matter would be used to search for the (3-D) topology of space [62].

(v) Other methods to note include $[23,24,26]$ specifically for the hyperbolic case and [27] for looking for images of our own galaxy as a quasar. In fact, a challenge to the astronomers and astrophysicists who try understand the dynamical, inter-stellar medium (ISM) and stellar past history of the Milky Way (MW) and Local Group galaxies (LG) is that they should be able to describe this history in a unique enough fashion such that high redshift galaxies can be excluded as possible topological images of the Milky Way just on the basis of intrinsic properties, rather than on checking for further topological images.

Since this school is intended for doctoral (graduate) students, it should be pointed out that this would provide a "safe" thesis project with relevance for cosmic topology, in that the main goal would be to understand the history of the MW and LG, which is already more than enough for a thesis. If it resulted that a "high" redshift galaxy (or a group) were found to have a striking resemblance to how the MW (or LG) should have looked at that epoch, then the student would not necessarily need to conclude that topological images have been found - the resemblance could be ascribed to coincidence and checked for cosmological significance during postdoctoral work (or by other researchers in the field). However, the advances in the understanding of the MW and LG history in order to make such a significant claim of resemblance would be sufficient for several publishable articles.

\subsubsection{Methods: two-dimensional}

The principle behind the use of the CMB, i.e. of a nearly two-dimensional thin shell from the observer's point of view, is that small portions of the shell corresponding to the CMB can be considered in some sense as objects. Depending on the topology of the Universe, some of these "objects" may occupy points of space which have topological images on other parts of the CMB. 


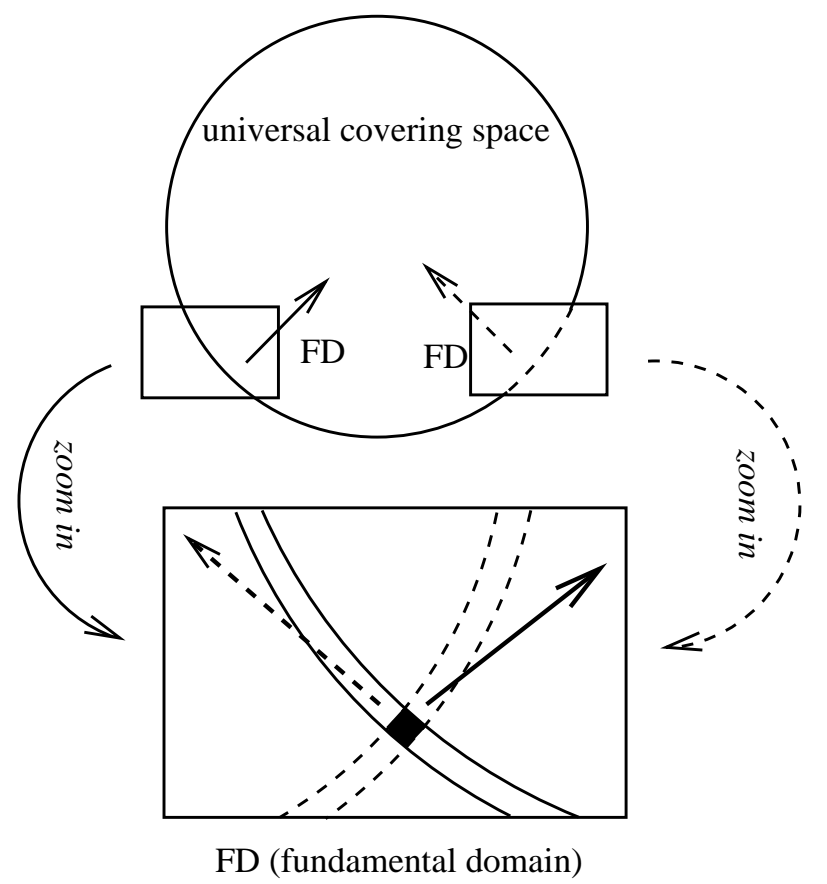

Figure 14. Schematic diagram showing basic principle of CMB methods. A single point of physical space emits radiation isotropically at the recombination epoch, but particularly in two different directions such that after crossing the Universe several times, the photons arrive at the observer. The upper half of the figure is from the point of view of the covering space, with several copies of the $F D$, while the lower half is a zoomed-in more physical point of view, in a single copy of the $F D$. The arrows indicate photons' paths. The "object" which is to be seen as multiple topological images is the intersection of the two thin sections of the shell of last scattering, i.e. the intersection shaded in black.

A schematic figure of this is shown in Fig. 14. For the "object", i.e. the intersection of two sections of the surface of last scattering (SLS), to be seen as multiple topological images with identical black-body temperatures, it has to have the same average temperature integrated in different directions, and any effects due to the time evolution across the SLS would have to be the same in the two directions (unless the two effects cancelled). In other words, the CMB radiation would have to be locally isotropic. This is expected to be the case on large scales for the naïve Sachs-Wolfe effect (gravitational redshift), but on medium scales $\left(\sim 1^{\circ}\right)$ the Doppler effect, which is not isotropic, is expected to dominate.

Another possible problem with CMB multiple imaging, which has not been explicitly analysed, is that the SLS is much thinner than the resolution of COBE, by a factor of $\sim 10^{2}$. That is, the black shaded intersection in Fig. 14 which is seen twice, is averaged with $\sim 10^{2}$ neighbouring patches in each of two directions perpendicular to one line of sight to give a single observed number, let us say in the direction of the solid arrow in the figure. The same black patch is also observed in the direction of the dashed arrow, but this time it is averaged with a set of $\sim 10^{2}$ patches in two directions perpendicular to the dashed arrow before the single observed value is obtained.

It is not clear whether or not these two averaging processes will lead to the same temperature fluctuation $\delta T / T$, even if the individual patches, un-observable individually, are dominated by the same physical process (gravitational potential). Averaging is a subtle process in physics, and given that the averaging in this case has different relevance depending on whether or not the hypothesis of simple-connectedness is adopted, it is not clear that reasoning in the former case is sufficient for the latter.

(i) The topology-independent method for using the CMB, supposing that these two problems 
can be overcome, is the "method of circles" $[10,86]$. Consider extending the tiling of the covering space by copies of the $F D$ beyond the observable sphere and consider the observable sphere of a second observer in one of the copies of the $F D$ outside the first sphere. The intersection of these two spheres is a circle.

But since each copy of the $F D$ is equivalent, the second observer can in fact perfectly well be physically identical with the first observer. That is, in the covering space, two observers, each of to whom the other is behind the CMB (but not too far), are equivalent to a single observer looking in two different directions towards the CMB, as long as they are at the same position within the $F D$.

Hence, the effect of multi-connectedness would be that the values of the temperature fluctuations $\delta T / T$ around certain circles on the $\mathrm{CMB}$ would map onto one another, since the circles correspond to the same set of points in space and time.

A related two-dimensional method to be applied to future satellites (MAP and Planck Surveyor), is that of searching for patterns of hot and cold spots [53]. This may bypass the problem of the large number of calculations to make for the circles method.

(ii) Other two-dimensional methods require (a) assuming the topology and specific quantitative parameters for individual 3-manifolds of each given topology and (b) modelling the power spectrum of perturbations. The latter is difficult to justify.

The theoretical motivation for Gaussian amplitude distributions, uniform random phases and a $P(k) \propto k^{1}$ power spectrum are unlikely to be valid at scales approaching $r_{\text {inj }}$ and $r_{+}$. That is, either for a hyperbolic or for a flat, $\lambda_{0} \sim 0.7$ metric to be presently observable, inflation needs some degree of fine-tuning (which can partly be provided by the ergodicity of geodesics in the former case, [8]). Since curvature or $\lambda_{0}>0$ must remain "uninflated" in the sense of being observable at the present epoch in these cases, it is unclear why perturbations on the scale of $R_{C} \sim R_{H}$ should necessarily be "inflated" in the sense of exactly satisfying the assumptions on the power spectrum. Moreover, even for other choices of metric, if the Universe is observably an MCM, then scales approaching $r_{\text {inj }}$ and $r_{+}$need not necessarily be "inflated" either.

The observational motivation for Gaussian amplitude distributions, uniform random phases and a $P(k) \propto k^{1}$ power spectrum are equally lacking for tests of MCM's. The only observational justification of these properties on large scales is COBE data, which is analysed assuming simpleconnectedness. A self-consistent assumption on the fluctuation spectrum for comparison of an MCM with COBE data would be to calculate this spectrum in three dimensions based on the COBE data shifted into the $F D$ of the MCM assumed. However, use of the resultant spectrum to simulate properties of the COBE observations would give ... exactly the COBE observations (if it has been done correctly).

So a self-consistent alternative to (b) would not enable rejection of an MCM. In other words, this approach tests assumptions on the the fluctuation spectrum (on the scale $\lesssim r_{\text {inj }}<r_{+}$) rather than the MCM itself. (Moreover, some authors find violation of Gaussian amplitude distributions in the COBE data [31, 60].)

Nevertheless, approach (ii) has been applied several times. Generally, a spherical harmonic analysis (" $C_{l}$ " spectrum) of simulated CMB maps (e.g. [78, 77]) is calculated.

For a hyperbolic spatial section, a standard Fourier analysis is, in a strict sense, invalid, whether or not a trivial topology is assumed. This compounds the problems of assumptions regarding the power spectrum, which cannot be defined in the usual way. Moreover, the classification and listing of the 3-manifolds is an open enough problem, and knowledge of eigenmodes for the equivalent of a Fourier analysis is even less developed. An interesting way to avoid this problem is to use correlation functions rather than power spectra for assumption (b). Bond, Pogosyan \& Souradeep [5] have done this for two of the many hyperbolic 3-manifolds known.

A general argument claimed for the methods which assume specific 3-manifolds is that no objects, i.e. no density fluctuations, which are larger than the Universe, i.e. larger than $r_{+}$, can 
exist, so there should be a cutoff at small $l$ values (large length scales) in the $C_{l}$ spectrum.

While this may sound common sense, it is only correct in the $F D$. In the universal covering space, fluctuations much larger than the Universe can indeed exist (e.g. $\sim 30$ times larger, see Fig. 1 of [65]). These structures cross the $F D$ many times. While the $C_{l}$ spectrum may indeed lose power on large scales due to averaging in different directions, it is incorrect to state that fluctuations larger than $r_{+}$cannot exist. The component eigenmodes in $3-\mathrm{D}$ are of course constrained by some integer multiple of $r_{+}$, but basis functions of the 2-D sphere cut to $\pm 20^{\circ}$ from the galactic plane only relate to the 3 -D eigenmodes via simplifying assumptions which once again may not be valid at these length scales in MCM's. Recently Inoue [40] computed the low-lying eigenmodes on some compact 3-hyperbolic spaces using the direct boundary element method, and found that the expansion coefficients behave as gaussian pseudo-random numbers.

A summary of the advantages and disadvantages of these different methods is shown in the first part of Table 2 .

Another interesting constraint may be derived when we take into account the possibility that phase transitions in the early universe could develop topological defects. Uzan \& Peter [84] showed that if space is multi-connected on scales now smaller than the horizon size, strings and domain walls were very unlikely to exist. Uzan [81, 82] generalized these results to textures and studied the cosmological implications of such constraints. He concluded that a large class of multi-connected universes with topological defects accounting for structure formation were ruled out by the cosmic microwave background observations.

\subsubsection{Constraints}

3-D (i,ii) The cosmic crystallography ([46], CC) and brightest cluster ([70], BC) methods indicate lower limits on the out-diameter of around $R_{H} / 20$ and $R_{H} / 10$ respectively, though the former is valid only in Euclidean spaces $\mathbf{R}^{3} / \Gamma$.

3-D (iii) The isometry search method applied to quasars is specifically designed to work for poor standard candles, so is intended for finding specific candidates for the 3-manifold, not for constraining $r_{\text {inj }}$ or $r_{+}$.

2-D (i) No application of the identified circles principle to COBE data to constrain $r_{\text {inj }}$ or $r_{+}$has yet been published, since the poor signal-to-noise and resolution make the chance of a detection seem unlikely.

2-D (ii) However, many applications of the two-dimensional methods to observations from the COBE satellite $[78,77,42,13,52,5]$ have been made. These can be interpreted either as suggesting constraints close to the horizon scale, but only for a (mostly Euclidean) finite number of the infinitely many topologies possible, or as showing that standard assumptions on the statistics of the perturbation spectrum on scales close to $r_{\text {inj }}$ and $r_{+}$would have to be wrong for one of these MCM's to be correct. Note that the confidence limits stated for several of these studies are statements about the distribution of random realisations, not about observational inconsistency of multiply imaged observed patches of the CMB.

These various direct constraints and perturbation spectrum based tests are listed in the second part of Table 2.

The poor resolution of $\mathrm{COBE}\left(\sim 10^{\circ}\right)$ implies that the data from the MAP and Planck satellites will probably be necessary before the circles principle can be applied seriously. This will not be easy, due to the Doppler effect, the integrated Sachs-Wolfe effect, other foregrounds, and the problem of averaging mentioned above.

\subsubsection{Specific candidates}

The most specific candidates suggested are those of [70] and [5], though an implicit candidate may also be implied by the application of the isometry search method to quasars [65]. 
The former candidate was noticed by chance in a small list of some of the brightest clusters chosen to illustrate the brightest cluster method. Among these seven clusters, three form a right angle to within 2\%-3\% accuracy (depending on the values of $\Omega_{0}$ and $\lambda_{0}$ ) and the two side lengths adjacent to the near-right angle are equal to within $1 \%$. This is better than tolerance considered for special quasar configurations by [27].

An a posteriori estimate of the probability of this occurring by chance would be difficult to make objectively, but since the configuration

(i) corresponds to that expected for the most commonly studied candidate in CMB studies (variations on hypertori),

(ii) implies generators which can easily be used to derive observationally refutable predictions, and

(iii) can be used to test in detail the validity of the different statistical methods,

it is useful to consider the implied $\widetilde{M} / \Gamma=T^{2} \times \mathbf{R}$ as a candidate 3 .

See [70] for object names, positions and values of the generators. A more recent summary of arguments for and against the candidate is provided in the discussion section of [68]; see also [66] for a COBE analysis. If the candidate were correct, then two topological images of the Coma cluster would be already known.

Bond et al. [5] (1998, §4.3) have also noticed a 3-manifold (for the two hyperbolic topologies considered) which, for a certain orientation, is "preferable to standard cold dark matter" when compared to the COBE observations. The volume of this manifold is slightly larger that that of the observable sphere, but $r_{\mathrm{inj}}$ is less than $R_{H}$, though not enough to imply any multiple topological images at observable sub-SLS distances.

\subsection{OTHER OBSERVATIONAL DIFFICULTIES}

Another observational complication, which has not generally been considered, particularly in the claims of constraints, i.e. lower limits on $r_{\text {inj }}$, is an important point by [22]: in a strict sense, it is already well established that we do not live in an FL universe. We live in what (locally, at least) is an almost FL universe. Density perturbations (humans, planets, stars, galaxies, large scale structure) exist.

Depending on the way in which space inside the observable sphere and the $F D$ differs from an exact FL universe, the real spatial geodesics will not correspond exactly to multiples (and sums) of the generators derived assuming an exact FL universe. Gravitational lensing is one example, though the effect is probably small.

\section{Conclusion}

The present surge of research in the mathematics, the physical theory and the observations of cosmological topology promise exciting developments in all three fields. Moreover, some of the spin-offs which would follow from a significant detection have been explicitly calculated: the measurement of transversal galaxy velocities [68], precise estimates of the metric parameters [71], position of the topological signature in the $\Omega_{0}-\lambda_{0}$ plane [83].

Mathematics: Continued work by mathematicians to understand and classify hyperbolic 3manifolds may help theorists develop ideas regarding the physics behind global topology, while further developments in the software (SNAPPEA) will be useful to observational astronomers.

Theory: The development of predictions by quantum gravity theories before a 3-manifold candidate is confirmed to high significance would be useful from an epistemological point of view. 
Observations: The numerous observational methods offer plenty of scope for improvements in the practical details while waiting for new surveys such as the SDSS (Sloan Digital Sky Survey) and new satellites such as XMM (X-ray Multiple Mission), MAP and Planck Surveyor.

However, it is not necessary to wait: specific candidates for the 3-manifold have been and should continue to be proposed. The benevolence of time allocation committees for the observational refutation (or strengthening) of the candidates should contribute significantly to observational cosmo-topology.

\section{References}

1. Atkatz, D., Pagels, H., 1982, Phys. Rv. D 25, 2065

2. Barbour J. \& Pfister H., 1995, (Eds.) Mach's Principle: From Newton's Bucket to Quantum Gravity (Einstein Studies, Vol 6), Birkhauser

3. Beardon, A.F. 1983, The Geometry of Discrete Groups (GTM91) New York:Springer

4. Bieberbach, L., 1911, Mathematische Annalen, 70,297. 1912; Ibid, 72, 400.

5. Bond J. R., Pogosyan D., Souradeep T., 1998, Class.Quant.Grav., 15, 2573 (astro-ph/9804041)

6. Briel U. G., Henry J. P., Böhringer H., 1992, A\&A, 259, L31

7. Carlip S., 1998, Class.Quant.Grav., 15, 2629 (gr-qc/9710114)

8. Cornish N. J., Spergel D. N., Starkman G. D., 1996, Phys.Rev.Lett., 77, 215

9. Cornish N. J., Spergel D. N., Starkman G. D., 1998a, astro-ph/9708225

10. Cornish N. J., Spergel D. N., Starkman G. D., 1998b, Class.Quant.Grav., 15, 2657 (astro-ph/9801212)

11. da Costa L. N., in Cosmic Velocity Fields, ed. Bouchet F., Lachièze-Rey M., (Gif-sur-Yvette, France: Editions Frontières), p.475

12. Dekel, A., Burnstein, D., White, S., 1996, in Critical Dialogues in Cosmology, ed. N.Turok, World Scientific.

13. de Oliveira Costa A., Smoot G. F., 1995, Ap.J., 448, 477

14. de Sitter W., 1917, MNRAS, 78, 3

15. Dowker H. F., Garcia R. S., 1998, Class.Quant.Grav., 15, 1859 (gr-qc/9711042)

16. Dowker H. F., Surya S., 1998, PhysRevD, 58, 124019 (gr-qc/9711070)

17. e Costa S. S., Fagundes H. V., 1998, gr-qc/9801066

18. Einstein, A., Postcard to Hermann Weyl, June 1918, from Einstein Archives, Princeton (freely translated from German by J.-P. Luminet).

19. Einstein, A., 1919, Postcard to Kelix Klein, April 16th, ibid.

20. Einstein, A., de Sitter W., 1932, Proc. Nat. Acad. Sci 18, 213.

21. Ellis, G.F.R. 1971, Gen.Rel.Grav. 2, 7

22. Ellis G.F.R., Schreiber, G., 1986, PhysLettA, 115, 97

23. Fagundes H. V., 1985, Ap.J., 291, 450

24. Fagundes H. V., 1989, Ap.J., 338, 618

25. H.V. Fagundes, 1993, Phys. Rev. Lett. 70, 1579

26. Fagundes H. V., 1996, Ap.J., 470, 43

27. Fagundes H. V., Wichoski U. F., 1987, Ap.J., 322, L5

28. Fagundes, H. \& Gausmann, E., 1997, On Closed Einstein-de Sitter Universes, astro-ph/9704259.

29. Fagundes, H. \& Gausmann, E., 1999, astro-ph/9811368

30. Feodoroff, E., 1885, Russian journal for crystallography and mineralogy, St Petersburg, 21.

31. Ferreira P. G., Magueijo J., Gorski K. M., 1998, Ap.J., 503, L1

32. Friedmann, A., 1922, Zeitschrift für Physik, 10, 37.

33. Friedmann, A., 1923, Mir kak prostranstvo i vremya (The Universe as Space and Time), Leningrad, Akademiya (French translation in Essais de Cosmologie, Le Seuil/Sources du Savoir, Paris, 1997.)

34. Friedmann A., 1924, Zeitschr.für Phys., 21, 326

35. Gabai, D. Meyerhoff, R., Thurston, N., 1996, Homotopy Hyperbolic 3-manifolds Are Hyperbolic, preprint available at http://www.msri.org/MSRI-preprints/online/1996-058.html

36. Gomero, G. I., Teixeira, A. F. F., Reboucas, M. J., Bernui, A., 1999, gr-qc/9811038

37. Gott J. R., 1980, MNRAS, 193, 153

1986, in Inner Space/Outer

38. Harrison, E., 1987, Darkness at night, Harvard University Press.

39. Hawking S., 1984, NuclPhysB, 239, 257

40. Inoue, K.T., astro-ph/9810034.

41. Ionicioiu R., 1998, gr-qc/9711069

42. Jing Y.-P., Fang L.-Z., 1994, PhysRevLett, 73, 1882 (astro-ph/9409072)

43. Klein, F., 1890, Mathematisches Annalen, 37, 544.

44. Killing, W., 1891, Mathematisches Annalen, 39, 257.

45. Lachièze-Rey M., Luminet J.-P., 1995, PhysRep, 254, 136

46. Lehoucq R., Luminet J.-P., Lachièze-Rey M., 1996, A\&A, 313, 339

47. Lehoucq R., Luminet J.-P., Uzan J.-Ph., 1999, A\&A, to be published (astro-ph/9811107) 
48. Lemaître, G., 1927, Ann. Soc. Sci. Bruxelles, ser. A, 47, 29.

49. Lemaître, G., 1931, MNRAS 90, 490.

50. Lemaître G., 1958, in La Structure et l'Evolution de l'Univers, Onzième Conseil de Physique Solvay, ed. Stoops R., (Brussels: Stoops), p.1

51. Lemaître, G., 1978 (posth.) in L'Univers, problème accessible à la science humaine, Revue d'Histoire Scientifique, 31, pp. 345-359.

52. Levin J., Scannapieco E., Silk J., 1998, Class.Quant.Grav., 15, 2689

53. Levin J., Scannapieco E., de Gasperis, G., Silk J., Barrow, J.D., 1998, astro-ph/9807206

54. Luminet, J.-P., 1997 L'invention du big bang, suivi de A. Friedmann, G. Lemaître : Essais de Cosmologie (Le Seuil/Sources du Savoir, Paris).

55. Luminet, J.-P., 1998, Acta Cosmologica, 24 (gr-qc/9804006)

56. Luminet, J.-P. \& Lachièze-Rey, M., 1994, La physique et l'infini (Flammarion/Dominos, Paris).

57. Madore J., Saeger L. A., 1998, Class.Quant.Grav., 15, 811 (gr-qc/9708053)

58. Matveev S.V., Fomenko A.T., 1988, Russian Math. Surveys 43, 3.

59. Novacki, W., 1934, Commentarii Mathematici Helvetici, 7, 81.

60. Pando, J., Valls-Gabaud, D., Fang, L.-Zh., 1998, PhysRevLett, accepted, (astro-ph/9810165)

61. Peebles, P.J.E., 1993, Principles of Physical Cosmology, Princeton, U.S.A.: Princeton Univ. Press

62. Pierre, M., 1999, Proceedings of the workshop Cosmological Topology in Paris 1998 (Obs. Paris/IAP, Paris 14 December 1998), http://www.iap.fr/users/roukema/CTP98/resumes.html

63. Rainer M., 1996, gr-qc/9602059.

64. Rosales J.-L., 1998, gr-qc/9712059

65. Roukema B. F., 1996, MNRAS, 283, 1147

66. Roukema B. F., 1999, submitted

67. Roukema, B. F. et al., 1999, Proceedings of the workshop Cosmological Topology in Paris 1998 (Obs. Paris/IAP, Paris 14 December 1998), http://www.iap.fr/users/roukema/CTP98/resumes.html

68. Roukema B. F., Bajtlik, S., 1999, submitted to MNRAS

69. Roukema B. F., Blanloeil V., 1998, Class.Quant.Grav., 15, 2645 (astro-ph/9802083)

70. Roukema B. F., Edge A. C., 1997, MNRAS, 292, 105

71. Roukema B. F., Luminet, J.-P., 1999, submitted

72. Schwarzschild, K. Vierteljahrschrift der Ast. Ges. 35 67, 1900

73. Schwarzschild, K., 1998, Class.Quant.Grav., 15, 2539 [english translation of Schwarzschild (1900)]

74. Spaans, M., 1999, gr-qc/9901025

75. Starkman G. D., 1998, Class.Quant.Grav., 15, 2529

76. Starkman, G. et al., 1998, Invited papers from the workshop Cosmology and topology (CWRU, Cleveland, 25-27 October 1997), Class. Quant. Grav. 15, 2529

77. Starobinsky A. A., 1993, JETPLett, 57, 622

78. Stevens D., Scott, Silk J., 1993, PhysRevLett, 71, 20

79. Thurston, W. P., 1979, The geometry and topology of three manifolds, (Princeton Lecture Notes)

80. Thurston, W. P., 1997, Three-Dimensional Geometry and Topology, ed. Levy, S., Princeton, U.S.A.: Princeton University Press

81. J-Ph. Uzan, 1998a, Phys. Rev. D58, 087301

82. J-Ph. Uzan, 1998b, Class. Quant. Grav. 15, 2711

83. Uzan J.-Ph., Lehoucq R., Luminet J.-P., 1999, in preparation

84. Uzan, J.-Ph., Peter P., 1997, Phys.Lett. B 406, 20

85. Weeks, J., 1985, PhD thesis, Princeton University.

86. Weeks J. R., 1998, Class.Quant.Grav., 15, 2599 (astro-ph/9802012)

87. Wolf, J.A., 1960, C. R. Acad. Si. Paris, 250, 3443.

88. Zel'dovich Y. B., Grishchuk L. P., 1984, MNRAS, 207, 23P 